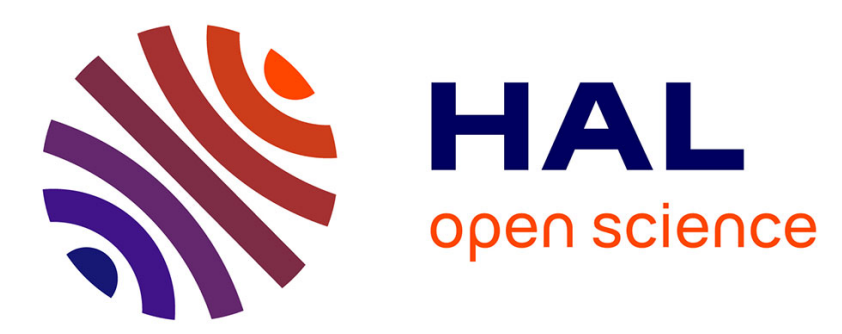

\title{
Propagation des ondes électromagnétiques dans les milieux présentant une perméabilité et une conductivité tensorielles
}

\author{
C. Monllor, M. Bouthinon, M. Baribaud
}

\section{- To cite this version:}

C. Monllor, M. Bouthinon, M. Baribaud. Propagation des ondes électromagnétiques dans les milieux présentant une perméabilité et une conductivité tensorielles. Revue de Physique Appliquée, 1978, 13 (7), pp.351-365. 10.1051/rphysap:01978001307035100 . jpa-00244460

\section{HAL Id: jpa-00244460 \\ https://hal.science/jpa-00244460}

Submitted on 1 Jan 1978

HAL is a multi-disciplinary open access archive for the deposit and dissemination of scientific research documents, whether they are published or not. The documents may come from teaching and research institutions in France or abroad, or from public or private research centers.
L'archive ouverte pluridisciplinaire HAL, est destinée au dépôt et à la diffusion de documents scientifiques de niveau recherche, publiés ou non, émanant des établissements d'enseignement et de recherche français ou étrangers, des laboratoires publics ou privés. 


\author{
Classification \\ Physics Abstracts \\ $76.50-72.30-75.30 \mathrm{D}-41.10 \mathrm{H}$
}

\title{
PROPAGATION DES ONDES ÉLECTROMAGNÉTIQUES DANS LES MILIEUX PRÉSENTANT UNE PERMÉABILITÉ ET UNE CONDUCTIVITÉ TENSORIELLES
}

\author{
C. MONLLOR, M. BOUTHINON et M. BARIBAUD
}

Laboratoire d'Electromagnétisme, E.N.S.E.R.G., 23, avenue des Martyrs, 38031 Grenoble, France

(Reçu le 30 décembre 1977, révisé le 4 avril 1978, accepté le 10 avril 1978)

\begin{abstract}
Résumé. - Après avoir rappelé brièvement les propriétés des ondes qui se propagent dans un milieu à perméabilité ou à conductivité tensorielle, nous étudions les matériaux qui présentent ces deux caractèrès simultanément. Les équations de Maxwell sont résolues pour des lames minces lorsque le champ magnétique statique est appliqué parallèlement ou perpendiculairement à leur plan. Ces deux configurations se rencontrent habituellement lors de la caractérisation des matériaux. Nous étudions également la propagation d'une onde guidée par le plan limitant un milieu semi-infini possédant un double caractère tensoriel. Cette structure d'onde est utilisée pour la réalisation de circuits hyperfréquences. Nous avons appliqué les résultats précédents à des lames de nickel dont la perméabilité et la conductivité tensorielles sont fonction du vecteur d'onde de l'onde qui s'y propage (dispersion spatiale). La connaissance de la répartition du champ électromagnétique à l'intérieur des échantillons permet une interprétation rigoureuse de tous les phénomènes observés (résonance ferromagnétique, ondes de spin stationnaire, singularités autour de l'antirésonance, etc.) aussi bien à température ambiante qu'à basse température.
\end{abstract}

\begin{abstract}
After a brief description of the properties of the waves propagating in a medium with a tensorial permeability or conductivity, we shall study the materials having these two characters simultaneously. Maxwell's equations are solved for thin plates when the static magnetic field is parallel or perpendicular to the plane of the plate. These two configurations generally appear when we characterize materials. We shall also study the propagation of a wave guided by the plane of a half boundless medium showing a double tensorial character. This wave structure has an application in the realization of microwave circuits. We have applied the preceding results to nickel plates in which tensorial permeability and conductivity depend on the propagating wave-vector (spatial dispersion). The knowledge of the electromagnetic field structure inside the samples allows us a precise interpretation of all the experimental results (ferromagnetic resonance, standing spin waves, pecularities near antiresonance, etc.) at room and at low temperatures.
\end{abstract}

Introduction. - La propagation des ondes électromagnétiques dans des matériaux aussi différents que les ferrites et les milieux conducteurs a été étudiée séparément par de nombreux auteurs. Nous allons montrer que la résolution des équations de Maxwell, le caractère des ondes qui s'y propagent et les phénomènes caractéristiques qui en découlent présentent de grandes similitudes. Par exemple, le traitement théorique de l'effet Faraday dans les milieux magnétiques est à rapprocher de celui de l'effet hélicon dans les conducteurs. De même, l'apparition des ondes de spin dans un matériau magnétique et des dopplerons dans un milieu conducteur se traitent d'une manière similaire en prenant en compte les effets de la dispersion spatiale respectivement dans les tenseurs de perméabilité et de conductivité. En plus de l'intérêt évident de tels rapprochements, cette démarche est rendue nécessaire lorsque l'on veut étudier la propagation dans des matériaux qui possèdent à la fois les caractères conducteur et magnétique tels que les semi-conducteurs ou conducteurs ferromagnétiques (nickel-permalloy). Ces travaux s'appliquent aussi bien à la caractérisation des matériaux qu'à leur application en vue de la conception de circuits en hyperfréquences. En présence d'un champ magnétique statique appliqué, ces matériaux présentent un caractère de gyrotropie magnétique ou électrique ou les deux simultanément. On peut alors les classer en trois catégories distinctes selon la conductivité de la substance : 
1) les isolants qui possèdent une perméabilité $\mu$ tensorielle; ce sont les milieux à gyrotropie magnétique comme les ferrites;

2) les conducteurs et semiconducteurs ordinaires qui présentent une conductivité $\sigma$ tensorielle et une perméabilité $\mu$ scalaire et constituent les milieux à gyrotropie électrique ;

3) enfin les matériaux à double gyrotropie dans lesquels la conductivité et la perméabilité sont tensorielles. C'est le cas le plus général dont font partie les conducteurs et les semiconducteurs ferromagnétiques.

Après avoir rappelé brièvement, dans la première partie, les principaux résultats obtenus avec une gyrotropie simple, nous allons étudier, dans la deuxième partie, le cas des milieux possédant un double caractère de gyrotropie. Dans la troisième partie, nous déterminerons la structure du champ électromagnétique dans une lame mince de nickel. Ceci nous permettra ensuite de décrire de façon détaillée et originale les phénomènes comme la résonance ferromagnétique, les ondes de spin stationnaires et l'antirésonance dans le nickel aussi bien à température ordinaire qu'à basse température.

1. Milieux à gyrotropie simple : perméabilité ou conductivité tensorielle. - 1.1 MILIEUX A GYROTROPIE MAGNÉTIQUE. - Lorsqu'on étudie la propagation d'ondes électromagnétiques de la forme $A \mathrm{e}^{j(\mathbf{k} . \mathbf{r}-\omega t)}$ dans une lame mince de matériau magnétiquement gyrotrope, aimanté à saturation par un champ magnétique $\mathbf{H}_{0}$ dirigé suivant $\mathrm{O} z$, la perméabilité dynamique $\boldsymbol{\mu}$ est tensorielle. Celle-ci est obtenue à partir de l'équation du mouvement de l'aimantation $\mathbf{M}$ et se présente sous la forme suivante :

$$
\boldsymbol{\mu}=\mu_{0}\left\|\begin{array}{lll}
\mu_{1} & j \mu_{2} & 0 \\
-j \mu_{2} & \mu_{1}^{\prime} & 0 \\
0 & 0 & \mu_{3}
\end{array}\right\| .
$$

Certains de ces matériaux présentent une propriété très intéressante en hyperfréquences : ils sont parfaitement isolants et se caractérisent par une permittivité dynamique scalaire $\varepsilon=\varepsilon_{0} \varepsilon_{\mathrm{r}}$. Ce sont les ferrites qui suscitent un grand intérêt dans la fabrication de dispositifs non réciproques. Lorsque des ondes planes se propagent dans de tels matériaux, les difficultés rencontrées lors de la résolution des équations de Maxwell proviennent du nombre des phénomènes physiques pris en compte qui sont traduits dans les coefficients $\mu_{i}$.

- Si l'on néglige les pertes, les effets du champ démagnétisant, la présence des ondes de spins et l'anisotropie magnétocristalline, on obtient la forme la plus simple introduite par Polder. Les expressions de $\mu_{i}$ dans lesquelles $\mu_{1}=\mu_{1}^{\prime}$ sont données par exemple en $[1 a]$.

- Les effets du champ démagnétisant lié à la forme des échantillons et éventuellement l'influence de l'anisotropie magnétocristalline compliquent notablement la résolution des équations de propagation [2]; ce qui conduit à des coefficients $\mu_{i}$ qui ne permettent une résolution analytique du problème que dans les deux cas particuliers de propagation parallèle et perpendiculaire représentés sur la figure 1 .

- La complexité du problème s'accroît encore lorsque l'onde électromagnétique provoque une variation de l'énergie d'échange qui se traduit par l'apparition des ondes de spins. Le milieu devient alors spatialement dispersif : la perméabilité est fonction $\mathrm{du}$ vecteur d'onde $\mathbf{k}$ de l'onde qui s'y propage.

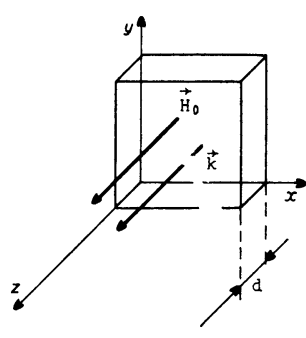

a)

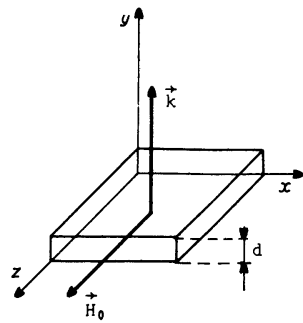

b)
FIG. 1. - $a$ ) propagation parallèle; $b$ ) propagation perpendiculaire.

[a) Parallel propagation; b) Perpendicular propagation.]

1.1.1 Propagation parallèle au champ magnétique. - Pour la géométrie représentée par la figure $1 a$ $\left(\mathbf{k} / / \mathbf{H}_{0}\right)$, nous supposons que les dimensions de l'échantillon dans le plan de la lame sont beaucoup plus grandes que son épaisseur $d$. Dans ces conditions, les axes $\mathrm{O} x$ et $\mathrm{O} y$ jouent des rôles analogues et $\mu_{1}^{\prime}=\mu_{1}$ si l'effet de l'anisotropie magnétocristalline peut être négligé. Lorsque cet effet intervient, on peut l'inclure dans la fréquence de résonance ferromagnétique $\omega_{\mathrm{r}}$ pour des lames taillées dans des plans [100] ou [111], [2]. Dans ces conditions, les coefficients du tenseur de perméabilité s'écrivent :

$$
\begin{aligned}
& \mu_{1}=\mu_{1}^{\prime}=1+\frac{\left(\omega_{\mathrm{r}}+\omega_{\mathrm{ech}} a^{2} k^{2}-j v_{\mathrm{m}}\right) \omega_{M}}{\left(\omega_{\mathrm{r}}+\omega_{\mathrm{ech}} a^{2} k^{2}-j v_{\mathrm{m}}\right)^{2}-\omega^{2}} \\
& \mu_{2}=\frac{-\omega_{M} \omega}{\left(\omega_{\mathrm{r}}+\omega_{\mathrm{ech}} a^{2} k^{2}-j v_{\mathrm{m}}\right)^{2}-\omega^{2}} \\
& \mu_{3}=1
\end{aligned}
$$

avec

- $\omega_{\mathrm{r}}$ : fréquence de résonance ferromagnétique : $\omega_{\mathrm{r}}=\gamma\left(H_{0}+H_{\mathrm{d}}+H_{\mathrm{a}}\right), \gamma=g e / 2 m_{0} c$ (C.G.S.) ou $\gamma=g e / 2 m_{0}$ (M.K.S.A.) ;

$g$ : facteur de décomposition spectrale, $H_{0}$ : champ magnétique appliqué,

$H_{\mathrm{d}}$ : champ démagnétisant,

$H_{\mathrm{a}}$ : champ magnétique d'anisotropie.

- $\omega_{\text {ech }}=\gamma H_{\text {ech }}:$ terme représentant le champ 
d'échange $H_{\text {ech }}$ qui se calcule à partir de la constante d'échange $A$ par :

$\omega_{\mathrm{ech}}=\gamma \frac{2 A}{M_{\mathrm{s}} a^{2}}$,

$M_{\mathrm{s}}$ : aimantation à saturation du matériau,

$a$ : longueur caractéristique de l'ordre de grandeur des dimensions de la maille élémentaire du réseau cristallin.

- $\omega_{M}=\gamma M_{\mathrm{s}}$

- $v_{\mathrm{m}}=1 / T$ : terme représentant les pertes dans l'équation de Bloch-Bloembergen et qui est lié au coefficient analogue $(\lambda)$ de l'équation de LandauLifschitz par l'expression :

$$
v_{\mathrm{m}}=\lambda \frac{\omega}{\omega_{M}} .
$$

La résolution des équations de Maxwell mène à une équation de dispersion qui fait apparaître les modes suivants [3] :

$$
k^{2}=\varepsilon_{\mathrm{r}} \frac{\omega^{2}}{c^{2}}\left(\mu_{1} \pm \mu_{2}\right) .
$$

Le signe $(+)$ est associé à une onde polarisée circulairement à gauche (dans le sens trigonométrique direct) pour un observateur regardant dans le sens de la propagation.

Le signe $(-)$ correspond à une polarisation circulaire droite. Une onde incidente à polarisation rectiligne se décompose en deux ondes contrarotatives qui se propagent à l'intérieur du matériau avec deux constantes de propagation différentes; la polarisation est ainsi modifiée au cours de la propagation : c'est l'effet Faraday.

1.1.2 Propagation perpendiculaire au champ magnétique. - a) Equations de dispersion. - Pour la géométrie représentée figure $1 b\left(\mathbf{k} \perp \mathbf{H}_{0}\right)$, la résolution des équations de Maxwell mène à une équation de dispersion qui fait apparaître les modes suivants [3] :

$$
\begin{aligned}
k^{2} & =\frac{\omega^{2}}{c^{2}} \varepsilon_{\mathrm{r}} \frac{\mu_{1} \mu_{1}^{\prime}-\mu_{2}^{2}}{\mu_{1}} \\
k^{2} & =\frac{\omega^{2}}{c^{2}} \varepsilon_{\mathrm{r}} .
\end{aligned}
$$

Les dimensions de l'échantillon dans le plan transversal à $\mathbf{H}_{0}$ ne sont pas identiques, $\mu_{1}$ et $\mu_{1}^{\prime}$ sont alors différents. Les valeurs de $\mu_{1}, \mu_{1}^{\prime}$ et $\mu_{2}$ dépendent de l'orientation du champ magnétique statique $\mathbf{H}_{0}$ par rapport aux axes cristallins de la lame [2].

b) Polarisation de ces modes. - Le mode représenté par (7) ne peut se propager que si le champ magnétique de l'onde d'excitation est dirigé parallèlement au champ statique $\mathbf{H}_{0}$. Dans ces conditions, l'aimantation alternative $\mathbf{m}$, située dans le plan transversal à $\mathbf{H}_{0}$ ne subit aucune sollicitation et le matériau se comporte comme un diélectrique. Ce mode est celui qui se propage en l'absence de champ magnétique statique; on l'appelle mode ordinaire.

L'autre mode représenté par (6), peut se propager si le champ magnétique de l'onde d'excitation n'est pas dirigé suivant $\mathrm{O} z$. Il possède une composante de champ magnétique dans la direction de propagation $\mathrm{O} x$ et son champ électrique est parallèle à $\mathrm{O} z$. C'est un mode transversal électrique TE que l'on appelle mode extraordinaire.

La composante longitudinale $h_{x}$ du champ magnétique alternatif $\mathbf{h}$ de ce mode devient prépondérante lorsque le vecteur d'onde $\mathbf{k}$ des ondes mises en jeu est grand. On a alors $\mathbf{k} \wedge \mathbf{h} \simeq 0(\operatorname{rot} \mathbf{h} \simeq 0)[1 b]$. Ce sont les modes magnétostatiques utilisés dans des configurations d'ondes de surface que nous mentionnerons au paragraphe 2.3.

Cette particularité ne se retrouve pas en propagation parallèle.

1.2 MilieuX a GYrotropie électriQue. - Dans un matériau conducteur, l'action d'un champ magnétique appliqué $\mathbf{H}_{0}$ se traduit par une conductivité dynamique tensorielle $\sigma$ que l'on exprime plus généralement par l'intermédiaire de la permittivité équivalente $\varepsilon$ reliée à la conductivité par l'expression :

$$
\boldsymbol{\varepsilon}=\varepsilon_{0}\left(\varepsilon_{\mathrm{r}} \mathbf{I}-\frac{\boldsymbol{\sigma}}{j \omega \varepsilon_{0}}\right) .
$$

Ce comportement est celui des métaux, semimétaux, semi-conducteurs ordinaires et de certains plasmas gazeux. Le tenseur $\varepsilon$ s'obtient à partir de l'équation cinétique des porteurs et s'écrit généralement de la façon suivante :

$$
\varepsilon=\varepsilon_{0}\left\|\begin{array}{lll}
\varepsilon_{1} & j \varepsilon_{2} & 0 \\
-j \varepsilon_{2} & \varepsilon_{1}^{\prime} & 0 \\
0 & 0 & \varepsilon_{3}
\end{array}\right\|
$$

La forme la plus simple des coefficients $\varepsilon_{i}$ se calcule à partir de la théorie classique de Drude-Zener qui suppose que tous les porteurs ont même vitesse. Dans ces conditions : $\varepsilon_{1}=\varepsilon_{1}^{\prime}$ [4].

La détermination de ces coefficients est plus compliquée si l'on tient compte de la répartition réelle des vitesses des porteurs. Il faut alors intégrer l'équation de Boltzmann sur la surface de Fermi [5]. Les valeurs de $\varepsilon_{1}$ et $\varepsilon_{1}^{\prime}$ peuvent être différentes selon le type de propagation envisagé (Fig. 1) même pour les conducteurs ayant une surface de Fermi sphérique [2]. D'autre part, il intervient dans les expressions de $\varepsilon_{i}$ des termes dépendant du vecteur d'onde $\mathbf{k}:$ le milieu devient là aussi spatialement dispersif.

Une difficulté supplémentaire apparaît dans les métaux portés à basse température. Le libre parcours moyen devient comparable à la longueur d'onde : c'est l'effet de peau anormal. Nous déterminerons au paragraphe 3.2.3 le seuil d'apparition de cet effet dans les conducteurs ferromagnétiques comme le nickel. 
1.2.1 Propagation parallèle au champ magnétique. - Lorsque $\mathbf{k}$ est parallèle à $\mathbf{H}_{0}$ (Fig. $\left.1 a\right), \varepsilon_{1}^{\prime}=\varepsilon_{1}$ et la résolution des équations de Maxwell mène à une équation de dispersion qui fait apparaître des modes à polarisations circulaires de sens opposés :

$$
k^{2}=\frac{\omega^{2}}{c^{2}}\left(\varepsilon_{1} \pm \varepsilon_{2}\right)
$$

avec :

$$
\varepsilon_{1}=1+\frac{\sigma_{0} v_{\mathrm{e}}\left(\omega+j v_{\mathrm{e}}\right)}{\varepsilon_{0} \omega\left(\left(v_{\mathrm{e}}-j \omega\right)^{2}+\omega_{\mathrm{c}}^{2}\right)} .
$$

Le terme unité représente le courant de déplacement que l'on peut négliger dans un bon conducteur

$$
\varepsilon_{2}=-\frac{\sigma_{0} v_{\mathrm{e}} \omega_{\mathrm{c}}}{\varepsilon_{0} \omega\left(\left(v_{\mathrm{e}}-j \omega\right)^{2}+\omega_{\mathrm{c}}^{2}\right)}
$$

où $\omega_{\mathrm{c}}=\mu_{0} \frac{e H_{0}}{m^{*}} ; m^{*}:$ masse effective des porteurs ; $v_{\mathrm{e}}$ : fréquence de collisions des porteurs; $\sigma_{0}$ : conductivité statique du matériau.

a) Dans le cas d'un métal bon conducteur à température ordinaire, on a : $\varepsilon_{2} \simeq 0$ et $\varepsilon_{1} \simeq-\sigma_{0} / j \omega \varepsilon_{0}$, le carré du vecteur d'onde est imaginaire pur : on retrouve l'effet de peau ordinaire.

b) Dans les semi-conducteurs, la fréquence cyclotronique $\omega_{\mathrm{c}}$ peut prendre des valeurs très grandes. Dans ces conditions, on ne peut plus négliger $\varepsilon_{2}$ devant $\varepsilon_{1}$; ce terme peut même devenir prépondérant ; l'équation de dispersion se ramène alors à :

$$
k_{ \pm}^{2}= \pm \frac{\omega^{2}}{c^{2}} \varepsilon_{2} .
$$

La solution $k_{-}$correspond aux ondes appelées hélicons par Aigrain. Ces ondes se propagent avec une très faible atténuation, car le courant de Hall lié à $\varepsilon_{2}$ est en quadrature avec le champ électrique $\mathbf{E}$ tandis que le courant de conduction, lié à $\varepsilon_{1}$ est en phase avec le champ électrique [6].

\subsubsection{Propagation perpendiculaire au champ magné-} tique. - Lorsque le vecteur d'onde $\mathbf{k}$ est perpendiculaire à $\mathbf{H}_{0}$ (Fig. 1b), l'équation de dispersion fait apparaître les modes suivants [3] :

$$
\begin{aligned}
& k^{2}=\frac{\omega^{2}}{c^{2}} \frac{\varepsilon_{1} \varepsilon_{1}^{\prime}-\varepsilon_{2}^{2}}{\varepsilon_{1}} \\
& k^{2}=\frac{\omega^{2}}{c^{2}} \varepsilon_{3} .
\end{aligned}
$$

Si l'on néglige le courant de déplacement dans un conducteur, l'équation (14) devient :

$$
k^{2}=i \frac{\omega}{c^{2}}\left(\sigma_{y y}-\frac{\sigma_{x y}^{2}}{\sigma_{x x}}\right) .
$$

C'est le mode extraordinaire analogue à celui défini au paragraphe 1.1.2. De la même façon, l'équation (15) peut s'écrire :

$$
k^{2}=j \omega \sigma_{0} \mu_{0} .
$$

C'est le vecteur d'onde de l'effet de peau ordinaire que nous appellerons par la suite $k_{\mathrm{e}}$.

2. Milieux à perméabilité et conductivité tensorielles. - Il existe un autre type de milieu qui présente à la fois un caractère de gyrotropie magnétique et de gyrotropie électrique. On est amené à étudier ce cas lorsqu'on s'intéresse à l'interprétation des phénomènes de propagation dans des échantillons de conducteurs ferromagnétiques comme le nickel ou de semi-conducteurs ferromagnétiques comme $\mathrm{CdCr}_{2} \mathrm{Se}_{4}$ soumis à l'action d'un champ magnétique statique.

2.1 DisPERSION DES ONDES EN PROPAGATION PARALLÈLE. - 2.1.1 Equation de dispersion. - Lorsque le champ magnétique appliqué $\mathbf{H}_{0}$ est perpendiculaire à la surface plane des échantillons, c'est-à-dire parallèle au vecteur d'onde $\mathbf{k}$ des ondes excitées (Fig. 1a), l'équation de dispersion s'écrit dans le cas le plus général :

$$
\begin{aligned}
& k^{4}-k^{2} \frac{\omega^{2}}{c^{2}}\left(\mu_{1} \varepsilon_{1}^{\prime}+\mu_{1}^{\prime} \varepsilon_{1}+2 \mu_{2} \varepsilon_{2}\right) \\
& \quad+\left(\mu_{1} \mu_{1}^{\prime}-\mu_{2}^{2}\right)\left(\varepsilon_{1} \varepsilon_{1}^{\prime}-\varepsilon_{2}^{2}\right) \frac{\omega^{4}}{c^{4}}=0 .
\end{aligned}
$$

Dans le cas où $\varepsilon_{1}^{\prime}=\varepsilon_{1}$ et $\mu_{1}^{\prime}=\mu_{1}$, cette équation se simplifie pour devenir :

$$
\begin{aligned}
& k_{\mathrm{G}}^{2}=\frac{\omega^{2}}{c^{2}}\left(\varepsilon_{1}+\varepsilon_{2}\right)\left(\mu_{1}+\mu_{2}\right) \\
& k_{\mathrm{D}}^{2}=\frac{\omega^{2}}{c^{2}}\left(\varepsilon_{1}-\varepsilon_{2}\right)\left(\mu_{1}-\mu_{2}\right) .
\end{aligned}
$$

On constate l'existence d'un couplage entre les propriétés électriques et magnétiques du milieu. Les modes solutions sont transversaux à polarisation circulaire gauche (17a) et droite (17b). Ce type d'onde se propage en particulier dans une lame de matériau conducteur ferromagnétique à surface de Fermi quasi sphérique $\left(\varepsilon_{1}=\varepsilon_{1}^{\prime}\right)$ et taillée dans des plans [100] ou [111] $\left(\mu_{1}=\mu_{1}^{\prime}\right)$ lorsque l'on tient compte de l'anisotropie magnétocristalline.

L'étude de ces modes s'avère nécessaire pour interpréter les résultats de nombreuses expériences réalisées sur des lames minces. Il faut alors tenir compte des pertes et de la dispersion spatiale. L'importance et la complexité du problème nécessitent un développement plus complet qui sera exposé au paragraphe 3. Nous nous limiterons dans l'immédiat au cas particulier négligeant les pertes et la dispersion spatiale. L'onde gauche (17a) offre peu d'intérêt, 
seule l'onde droite $(17 b)$ présente des singularités lorsque le champ magnétique statique varie; son équation de dispersion se ramène à :

$$
k_{\mathbf{D}}^{2}=\frac{\omega^{2}}{c^{2}}\left(1-\frac{\omega_{\mathbf{p}}^{2}}{\omega\left(\omega-\omega_{\mathrm{c}}\right)}\right)\left(1+\frac{\omega_{M}}{\omega_{\mathrm{r}}-\omega}\right)
$$

avec $\omega_{\mathrm{p}}$ : pulsation plasma $=\sqrt{\sigma_{0} v_{\mathrm{e}} / \varepsilon_{0}}$.

Le dernier terme représente la perméabilité effective ; il devient infini pour la fréquence $\omega_{\mathrm{r}}$ et s'annule pour $\omega_{\mathrm{a}}=\omega_{\mathrm{r}}+\omega_{M}$. Lorsque la fréquence $\omega$ est égale à la fréquence de résonance ferromagnétique $\omega_{\mathrm{r}}$, le vecteur d'onde $\mathbf{k}$ est très grand. Dans ces conditions, tous les spins sont parallèles au champ magnétique hyperfréquence. C'est la précession uniforme bien connue. Par contre, si $\omega$ est égale à la fréquence $\omega_{a}$, les spins restent parallèles entre eux mais sont opposés au champ magnétique alternatif. Cette situation particulière est généralement appelée antirésonance.

2.2.2 Milieux à perméabilité et permittivité effectives négatives. - Si la masse effective des porteurs $m^{*}$ est inférieure à la masse de l'électron $m_{0}$, on a :

$$
\omega_{\mathbf{c}}>\omega_{\mathbf{a}}>\omega_{\mathbf{r}}
$$

et l'équation (18) permet d'établir les courbes de dispersion représentées sur la figure $2 a$.

La figure $2 b$ donne la courbe de dispersion des
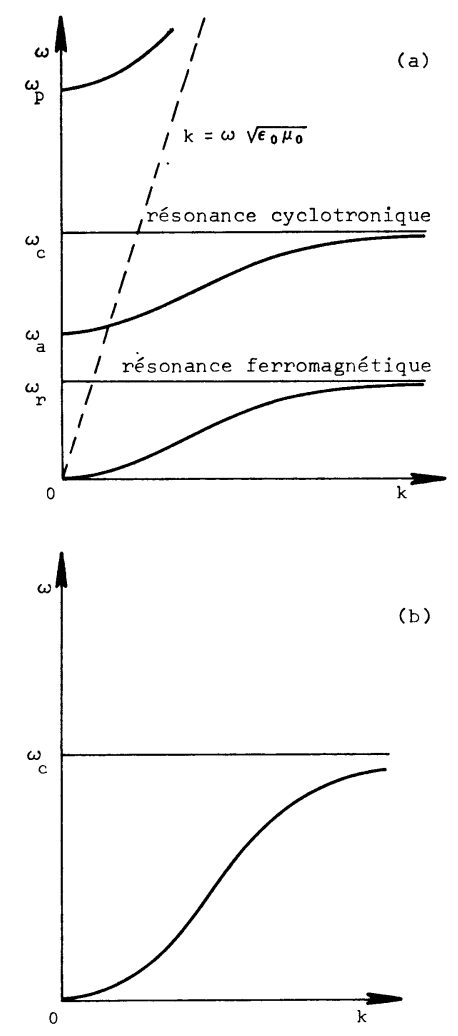

FIG. 2. - Courbes de dispersion dans un milieu conducteur lorsque la masse effective des porteurs est inférieure à la masse de l'électron libre; $a$ ) milieu magnétique ; $b$ ) milieu non magnétique. [Dispersion curve in a conductive medium when the effective mass of the carriers is smaller than the mass of the free electron. a) Non magnetic medium ; b) Magnetic medium.] hélicons dans un milieu conducteur non magnétique. La comparaison des figures $2 a$ et $2 b$ montre que dans les milieux ferromagnétiques conducteurs, au voisinage de l'antirésonance, la propagation peut s'effectuer avec des vecteurs d'ondes beaucoup plus faibles que ceux correspondant aux hélicons pour un même champ magnétique appliqué.

- Une situation très particulière et spécifique de matériaux à la fois conducteurs et ferromagnétiques peut apparaître lorsque la masse effective $m^{*}$ est supérieure à la masse de l'électron $m_{0}$. L'ordre des pôles et des zéros de l'équation de dispersion est alors inversé et mène aux résultats représentés sur la figure 3.

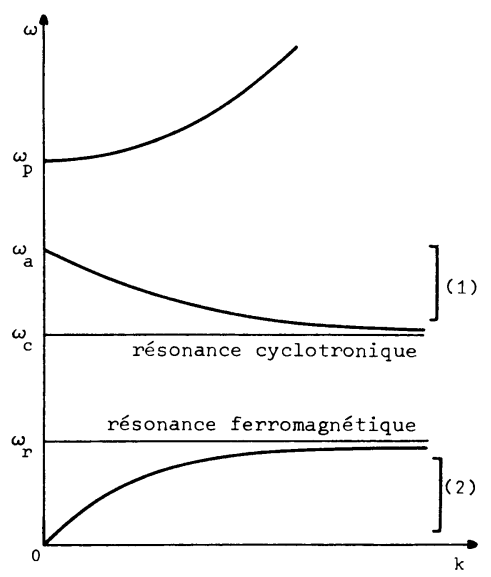

Fig. 3. - Courbes de dispersion dans un milieu conducteur ferromagnétique lorsque la masse effective des porteurs est supérieure à la masse de l'électron libre. (1) : $\varepsilon_{\text {eff }}=\varepsilon_{0}\left(\varepsilon_{1}-\varepsilon_{2}\right)<0$; $\mu_{\text {eff }}=\mu_{0}\left(\mu_{1}-\mu_{2}\right)<0 ; \quad$ (2) $\varepsilon_{\text {eff }}=\varepsilon_{0}\left(\varepsilon_{1}-\varepsilon_{2}\right)>0$; $\mu_{\text {eff }}=\mu_{0}\left(\mu_{1}-\mu_{2}\right)>0$.

[Dispersion curve in a ferromagnetic conductive medium when the effective mass of the carriers is greater than the mass of the free electron.]

La loi de dispersion de ces modes peut toujours se mettre sous la forme :

$$
k^{2}=\frac{\omega^{2}}{c^{2}} \varepsilon_{\mathrm{eff}} \mu_{\mathrm{eff}} .
$$

Lorsque l'une des grandeurs ( $\varepsilon_{\text {eff }}$ ou $\mu_{\text {eff }}$ ) est négative, il n'y a pas de propagation. Nous remarquons toutefois sur la figure 3 que, dans la plage de pulsations comprises entre $\omega_{\mathrm{c}}$ et $\omega_{\mathrm{a}}$, les deux quantités $\varepsilon_{\text {eff }}$ et $\mu_{\text {eff }}$ sont simultanément négatives et par conséquent la propagation d'ondes y est possible. Ce type de propagation ne peut se manifester que dans des milieux à perméabilité et conductivité tensorielles. Ceci doit conduire à des propriétés bien spécifiques, apparemment étonnantes comme l'ont déjà mentionné théoriquement Veselago [7] ainsi que Blank et Kaganov [8].

2.2 DisPERSION DES ONDES EN PROPAGATION PERPENDICULAIRE. - 2.2.1 Equations de dispersion. Lorsque le champ magnétique appliqué $\mathbf{H}_{0}$ est 
parallèle à la surface plane des échantillons, c'est-àdire perpendiculaire au vecteur d'onde $\mathbf{k}$ des ondes excitées (Fig. 1b), les équations de dispersion admettent les solutions suivantes dans lesquelles les caractères magnétique et électrique du milieu interviennent séparément :

$$
\begin{aligned}
k_{\mathrm{em}}^{2} & =\frac{\omega^{2}}{c^{2}} \frac{\varepsilon_{3}}{\mu_{1}}\left(\mu_{1} \mu_{1}^{\prime}-\mu_{2}^{2}\right) \\
k_{\mathrm{ee}}^{2} & =\frac{\omega^{2}}{c^{2}} \frac{\mu_{3}}{\varepsilon_{1}}\left(\varepsilon_{1} \varepsilon_{1}^{\prime}-\varepsilon_{2}^{2}\right) \\
\varepsilon_{3} & =0 .
\end{aligned}
$$

Le mode représenté par (20c) correspond aux ondes de plasma et se situe, en général au-dessus du domaine de fréquences correspondant aux micro-ondes. Par analogie avec ce qui a été dit précédemment, le mode $k_{\text {em }}$ peut être appelé mode extraordinaire magnétique : c'est un mode TE. Le mode $k_{\text {ee }}$ peut être appelé mode extraordinaire électrique : c'est un mode TM. Pour ce dernier, en négligeant le courant de Hall associé à $\varepsilon_{2}$, on retrouve le mode de l'effet de peau ordinaire du vecteur d'onde $k_{\mathrm{e}}$ tel que :

$$
k_{\mathrm{e}}^{2}=\left(\frac{1+j}{\delta}\right)^{2}=j \omega \mu_{0} \sigma_{0}
$$

où $\delta$ est l'épaisseur de peau classique du milieu conducteur.

Pour exciter le mode TE, le champ magnétique dans le plan transversal doit être parallèle à l'axe $O x$ (Fig. 1b). C'est la géométrie utilisée par Ament et Rado [9] pour étudier ce qu'ils appelèrent les résonances d'ondes de spin dans les métaux ferromagnétiques. Il s'agit, en fait, de l'étude de l'élargissement de la raie de résonance ferromagnétique causée par la présence des ondes de spin. Ces ondes sont caractérisées par le terme $\omega_{\text {ech }} a^{2} k^{2}$ dans les coefficients du tenseur de perméabilité. Afin de confronter nos résultats avec ceux obtenus par ces auteurs, nous adopterons momentanément leurs variables :

$$
\begin{gathered}
\Omega=\frac{\omega}{4 \pi M_{\mathrm{s}} \gamma}=\frac{\omega}{\omega_{M}} ; \quad \eta=\frac{H_{0}}{4 \pi M_{\mathrm{s}}}=\frac{\gamma H_{0}}{\omega_{M}} ; \\
\varepsilon^{2}=\frac{A}{2 \pi M_{\mathrm{s}}^{2} \delta^{2}}=\frac{\omega_{\mathrm{ech}} a^{2}}{\omega_{M} \delta^{2}} ; \\
K^{2}=k^{2} \varepsilon^{2} \delta^{2}=k^{2} \frac{\omega_{\mathrm{ech}} a^{2}}{\omega_{M}} ; L=\frac{\lambda}{\gamma M_{\mathrm{s}}}=\frac{v_{\mathrm{m}}}{\omega} .
\end{gathered}
$$

En reportant les valeurs de $\mu_{1}, \mu_{1}^{\prime}$ et $\mu_{2}$ dans l'équation $(20 a)$, on retrouve l'équation de dispersion de Ament et Rado :

$$
K^{6}-C_{1} K^{4}+C_{2} K^{2}-C_{3}=0 .
$$

Cette équation admet comme solutions :

- trois modes progressifs se propageant vers les $y$ positifs,
- trois modes régressifs se propageant vers les $y$ négatifs.

L'équation (20a) peut se mettre sous la forme :

$$
k^{2}=k_{\mathrm{e}}^{2}\left(\mu_{1}^{\prime}-\frac{\mu_{2}^{2}}{\mu_{1}}\right) \text {. }
$$

La quantité $\left(\mu_{1}^{\prime}-\frac{\mu_{2}^{2}}{\mu_{1}}\right)$ correspond à la perméabilité équivalente d'Ament et Rado. Avec les approximations de ces auteurs, on montre que :

$\mu_{\mathrm{eq}}=\mu_{1}^{\prime}-\frac{\mu_{2}^{2}}{\mu_{1}}=\frac{\eta-\Omega^{2}+j \Omega L+2 \varepsilon(1+j)}{\left(\eta-\Omega^{2}+j \Omega L+\varepsilon(1+j)\right)^{2}}$.

L'impédance de surface calculée à partir de cette théorie s'obtient en remplaçant $\mu_{0}$ par $\mu_{\text {eq }}$ dans l'impédance de surface d'un métal ordinaire. Les approximations faites supposent que $\eta, \Omega^{2}$ et $L^{2}$ sont beaucoup plus petits que l'unité. Dans le nickel, cette hypothèse est justifiée jusqu'à des fréquences de l'ordre du gigahertz, mais n'est plus valable au-delà. Ceci peut expliquer que de nombreux résultats d'expériences effectuées à qúelques dizaines de gigahertz n'aient pu être interprétés avec de telles approximations.

2.3 ONDES GUIDÉES PAR UN DIOPTRE PLAN (ONDES DE SURFACE). - Les deux situations géométriques précédentes sont principalement utilisées en vue de la caractérisation des matériaux. Les échantillons sont alors étudiés en réflexion ou en transmission.

La structure représentée sur la figure $4 a$ est utilisée
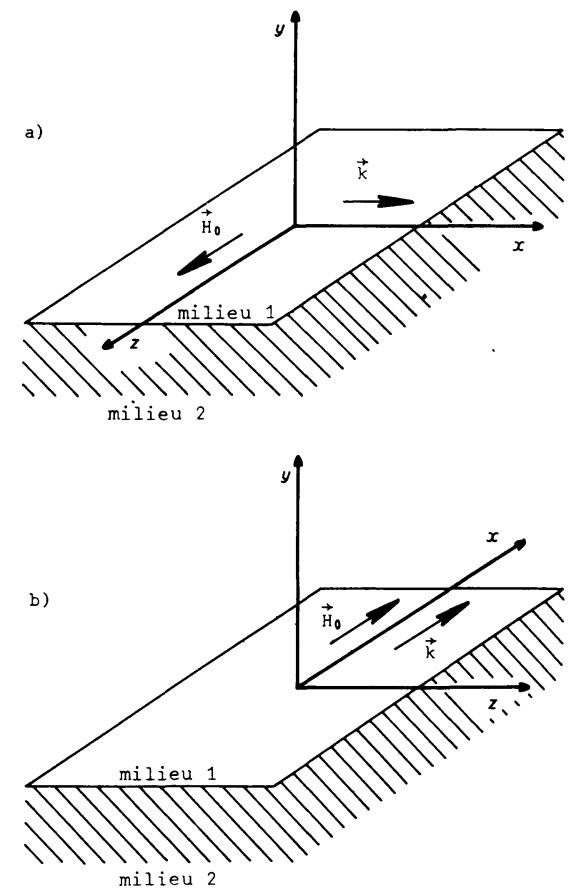

FIG. 4. - Propagation d'ondes de surface (champ magnétique dans le plan de séparation des deux milieux) ; $a$ ) $\mathbf{k}$ perpendiculaire à $\left.\mathbf{H}_{0} ; b\right) \mathbf{k}$ parallèle à $\mathbf{H}_{0}$.

[Surface wave propagation (magnetic field in the boundary plane between the two mediums).] 
pour la réalisation de circuits hyperfréquences comme les isolateurs [10-12] ou éventuellement des amplificateurs [13-14] dans des guides ou des lignes microrubans. Les autres géométries (Fig. $4 b$ et 5) dont l'étude est plus complexe ont également été proposées pour réaliser de tels circuits [15-16]. Nous allons simplement montrer dans ce paragraphe que les modes qui se propagent dans de telles structures peuvent être obtenus par une combinaison des modes propres étudiés précédemment.

2.3.1 Champ magnétique $\mathbf{H}_{0}$ dans le plan de séparation (Fig. 4a). - L'onde se propage selon Ox. Les amplitudes complexes des composantes suivant Ox des vecteurs d'onde dans les milieux 1 et 2 sont identiques dans le plan de séparation de ces deux milieux, soit : $k_{1 x}=k_{2 x}=k$.

On considère qu'il n'y a aucune propagation suivant la direction $\mathrm{O} z$. Les expressions des champs sont de la forme :

- dans le milieu $1: A_{1} \mathrm{e}^{j\left[k_{x} x+\gamma_{1} y-\omega t\right]}$;

- dans le milieu $2: A_{2} \mathrm{e}^{j\left[k_{x} x+\gamma_{2} y-\omega t\right]}$.

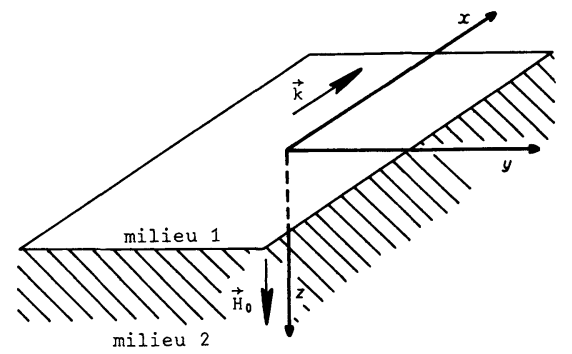

FIG. 5. - Propagation d'ondes de surface (champ magnétique perpendiculaire au plan de séparation des deux milieux).

[Surface wave propagation (magnetic field perpendicular to the boundary plane between the two mediums).]

Si le milieu 1 est l'air et le milieu 2 un matériau à perméabilité et conductivité tensorielles, la résolution des équations de Maxwell mène aux équations de dispersion suivantes :

Pour le milieu 1 :

$$
k_{x}^{2}+\gamma_{1}^{2}=\frac{\omega^{2}}{c^{2}} .
$$

Pour le milieu 2 :

$$
k_{x}^{2}+\gamma_{2}^{2}=\frac{\omega^{2}}{c^{2}} \varepsilon_{3}\left(\frac{\mu_{1}^{2}-\mu_{2}^{2}}{\mu_{1}}\right)
$$

ou :

$$
k_{x}^{2}+\gamma_{2}^{2}=\frac{\omega^{2}}{c^{2}} \mu_{3}\left(\frac{\varepsilon_{1}^{2}-\varepsilon_{2}^{2}}{\varepsilon_{1}}\right) \text {. }
$$

On retrouve les modes propres de la propagation perpendiculaire. Ce résultat était prévisible car les deux directions de propagation (Ox et $\mathrm{O} y$ ) sont toutes deux perpendiculaires au champ appliqué $\mathbf{H}_{0}$. Les modes TE (24b) et TM (24c) ne peuvent pas être couplés dans un même milieu car ils mettent en jeu des composantes de champ différentes. Pour obtenir un couplage entre les propriétés électriques et magnétiques, il faut, par exemple, que le milieu 1 soit constitué d'un matériau semi-conducteur ordinaire et le milieu 2 d'un ferrite.

Sur une lame de ferrite, seul le mode extraordinaire magnétique se propage. Il a été utilisé pour réaliser des lignes unidirectionnelles [10-11]. La composante longitudinale magnétique de ce mode peut devenir prépondérante : c'est alors un mode magnétostatique qui trouve son application dans l'étude des lignes à retard [17].

2.3.2 Champ magnétique $\mathbf{H}_{0}$ perpendiculaire au plan de séparation (Fig. 5). - Dans le milieu 1, la relation de dispersion des ondes électromagnétiques est identique à la relation (24a). Par contre, dans le milieu 2, la résolution des équations de Maxwell est plus compliquée que dans le cas précédent. La propagation s'effectuant suivant $\mathrm{O} x$, c'est-à-dire perpendiculairement à $\mathbf{H}_{0}$, met en jeu les modes extraordinaires électrique et magnétique $k_{2 \mathrm{e}}$ et $k_{2 \mathrm{~m}}$. De la même façon, la propagation selon $\mathrm{O} z$ (parallèlement à $\mathbf{H}_{0}$ ) fait intervenir les modes droit et gauche $k_{2 \mathrm{D}}$ et $k_{2 \mathrm{G}}$ qui couplent les propriétés électriques et magnétiques du milieu. Dans le cas général, la propagation s'effectuant à la fois selon $\mathrm{O} x$ et selon $\mathrm{O} y$, l'équation de dispersion peut se mettre sous la forme :

$$
\begin{aligned}
\left(\gamma_{2}^{2}-k_{2 \mathrm{G}}^{2}\right) & \left(\gamma_{2}^{2}-k_{2 \mathrm{D}}^{2}\right)+k^{2} \gamma^{2}\left(\frac{\mu_{1}}{\mu_{3}}+\frac{\varepsilon_{1}}{\varepsilon_{3}}\right) \\
& +\frac{\varepsilon_{1} \mu_{1}}{\varepsilon_{3} \mu_{3}} k^{2}\left(k^{2}-\left(k_{2 \mathrm{~m}}^{2}+k_{2 \mathrm{e}}^{2}\right)\right)=0 .
\end{aligned}
$$

La structure représentée sur la figure $4 b$ offre la même particularité et mène à une équation de dispersion analogue en permuttant les rôles respectifs de $k$ et $\gamma_{2}$.

2.4 INTÉR TS DES MILIEUX A PERMÉABILITÉ ET CONDUCTIVITÉ TENSORIELLES. - D'après l'étude qui précède, il est théoriquement possible de transposer les effets de propagation obtenus dans des ferrites aux semi-conducteurs ordinaires. Des circuits hyperfréquences [18-19] et plus précisément un isolateur à lame d'InSb fonctionnant entre 80 et $110 \mathrm{GHz}$ avec un champ magnétique de l'ordre de 15000 gauss [20] ont déjà été réalisés. Avec des champs aussi faibles, on ne peut pas obtenir des performances analogues avec des ferrites. Théoriquement, les semi-conducteurs ferromagnétiques offrent à la fois l'intérêt des semiconducteurs ordinaires et des ferrites. Des matériaux de ce type sont actuellement disponibles (EuO, $\mathrm{CdCr}_{2} \mathrm{Se}_{4}, \mathrm{CdCr}_{2} \mathrm{~S}_{4}$, etc.). Ils présentent en général une aimantation à saturation convenable mais les températures de Curie sont inférieures à l'ambiante. Leur gros inconvénient provient surtout de leur faible mobilité qui est beaucoup plus petite que celle de l'InSb [21-22]. Devant ces difficultés on s'est 
orienté vers des structures mixtes semi-conducteurs ordinaires ferrites pour la conception de circuits hyperfréquences. Mais on assiste actuellement à un nouvel essor du développement industriel de matériaux semi-conducteurs ferromagnétiques [23, 24].

Le nickel constitue actuellement l'un des matériaux qui présentent en hyperfréquences des effets liés au double caractère tensoriel. Bien qu'il ne soit pas susceptible d'applications dans les circuits en ondes centimétriques, il a été très largement étudié en vue de l'interprétation de phénomènes de propagation fondamentaux.

3. Etude de la propagation parallèle ; couplage des ondes de spin et des ondes électrocinétiques.- - $\mathrm{La}$ propagation parallèle $a$ été très souvent utilisée pour l'observation des phénomènes suivants :

- résonance ferromagnétique,

- résonance stationnaire d'ondes de spin,

- transmission autour de l'antirésonance,

- phénomènes résonnants dus au couplage des ondes de plasma et des ondes de spin qui se produisent à basse température et que nous avons introduit récemment [25]. Ces expériences ont été proposées pour déterminer les différents paramètres caractéristiques des matériaux, soient :

- aimantation à saturation $M_{\mathrm{s}}$,

- rapport gyromagnétique $\gamma$,

- constante d'échange $A$,

- facteur de pertes $\lambda$ de l'équation de LandauLifschitz ou $1 / T=v_{\mathrm{m}}$ de l'équation de Bloch-Bloembergen,

- constantes d'anisotropie $K_{1}, K_{2}, K_{3}$,

- constante d'anisotropie de surface $K_{\mathrm{s}}$ qui intervient dans le blocage plus ou moins complet des spins en surface.

Parmi les phénomènes résonnants cités ci-dessus, l'observation des ondes de spin stationnaires dans des échantillons de permalloy ou de nickel a donné lieu à un nombre impressionnant de publications (plus de neuf cents déjà répertoriées en 1973 [26]) destinées le plus souvent à proposer des théories permettant d'expliquer les résultats expérimentaux. Dans cette optique, nous avons été amenés à mettre au point un modèle théorique qui tient compte de l'influence des phénomènes de transport sur la propagation des ondes de spins. Pour exposer ce modèle, nous examinerons successivement les deux cas suivants qui constituent chacun une étape dans l'ordre de difficulté croissante de la résolution des équations de Maxwell :

1) à température ordinaire, la conductivité effective ne dépend pas de $k$ et le milieu n'est donc spatialement dispersif que par sa perméabilité $\mu_{i j}(k)$ du fait de la présence des ondes de spin;

2) à basse température, la conductivité dépend aussi de $k$ : le milieu présente un double caractère de dispersion spatiale.
3.1 Couplage DeS ONDES ÉleCtrocinÉTIQueS ET DES ONDES DE SPIN A TEMPÉRATURE ORDINAIRE. 3.1.1 Existence du couplage. - Si l'on tient compte des pertes et de la dispersion spatiale du tenseur de perméabilité, l'équation générale de dispersion (17b) donnant l'amplitude complexe du vecteur d'onde pour les modes droits s'écrit :

$$
\begin{aligned}
k^{2}=\frac{\omega^{2}}{c^{2}} & \left(\frac{\omega_{\mathrm{p}}^{2}\left(\omega+j v_{\mathrm{e}}+\omega_{\mathrm{c}}\right)}{\omega\left(\left(v_{\mathrm{e}}-j \omega\right)^{2}+\omega_{\mathrm{c}}^{2}\right)}\right) \times \\
& \times\left(1+\frac{\omega_{M}}{\omega_{\mathrm{r}}+\omega_{\mathrm{ech}} a^{2} k^{2}-j v_{\mathrm{m}}-\omega}\right) .
\end{aligned}
$$

Pour simplifier l'écriture de cette équation, nous poserons :

$$
k_{\mathrm{e}}^{2}=\frac{\omega^{2}}{c^{2}}\left(\frac{\omega_{\mathrm{p}}^{2}\left(\omega+j v_{\mathrm{e}}+\omega_{\mathrm{c}}\right)}{\omega\left(\left(v_{\mathrm{e}}-j \omega\right)^{2}+\omega_{\mathrm{c}}^{2}\right.}\right) .
$$

C'est l'amplitude complexe du vecteur d'onde qui existe dans un métal fictif ayant les mêmes propriétés de conduction mais n'en possédant pas le caractère magnétique.

Lorsque la température augmente et que les collisions deviennent prépondérantes, $k_{\mathrm{e}}$ s'identifie avec le vecteur d'onde de l'effet de peau ordinaire. En définissant $k_{\mathrm{m}}$ par l'expression :

$$
\omega=\omega_{\mathrm{r}}-j v_{\mathrm{m}}+\omega_{\mathrm{ech}} a^{2} k_{\mathrm{m}}^{2}
$$

l'expression (26) devient :

$$
\left(k^{2}-k_{\mathrm{e}}^{2}\right)\left(k^{2}-k_{\mathrm{m}}^{2}\right)=\frac{k_{\mathrm{e}}^{2} \omega_{M}}{\omega_{\mathrm{ech}} a^{2}} .
$$

Cette équation exprime clairement l'existence d'un couplage entre les ondes de spins caractérisées par le vecteur d'onde $k_{\mathrm{m}}$ et les ondes caractérisées par le vecteur d'onde $k_{\mathrm{e}}$ que nous appellerons ondes électrocinétiques en généralisant la définition donnée par Steele et Vural [4]. Ces auteurs ont ainsi nommé dans un conducteur les ondes dont les propriétés dépendent du mouvement des porteurs. Ainsi, l'effet de peau ordinaire est caractérisé par une onde électrocinétique où le mouvement provient des collisions.

Afin d'étudier ce couplage par la suite, nous pouvons mettre l'équation (29) sous la forme :

$$
k^{4}-k^{2}\left(k_{\mathrm{e}}^{2}+k_{\mathrm{m}}^{2}\right)+k_{\mathrm{e}}^{2} k_{\mathrm{m}}^{2}(1-\eta)=0
$$

en définissant le facteur de couplage $\eta$ par :

$$
\eta=\frac{\omega_{M}}{\omega-\omega_{\mathrm{r}}+j v_{\mathrm{m}}} .
$$

3.1.2 Modes d'ondes de spins. - Les solutions de l'équation de dispersion (30) s'écrivent :

$$
\begin{aligned}
k_{1}^{2} & =\frac{1}{2}\left(k_{\mathrm{e}}^{2}+k_{\mathrm{m}}^{2}+\left(k_{\mathrm{e}}^{2}-k_{\mathrm{m}}^{2}\right)\left(1+\eta \frac{4 k_{\mathrm{e}}^{2} k_{\mathrm{m}}^{2}}{\left(k_{\mathrm{e}}^{2}-k_{\mathrm{m}}^{2}\right)^{2}}\right)^{1 / 2}\right) \\
& =\left(\alpha_{1}+j \beta_{1}\right)^{2}
\end{aligned}
$$




$$
\begin{aligned}
k_{2}^{2} & =\frac{1}{2}\left(k_{\mathrm{e}}^{2}+k_{\mathrm{m}}^{2}-\left(k_{\mathrm{e}}^{2}-k_{\mathrm{m}}^{2}\right)\left(1+\eta \frac{4 k_{\mathrm{e}}^{2} k_{\mathrm{m}}^{2}}{\left(k_{\mathrm{e}}^{2}-k_{\mathrm{m}}^{2}\right)^{2}}\right)^{1 / 2}\right) \\
& =\left(\alpha_{2}+j \beta_{2}\right)^{2} .
\end{aligned}
$$

Les constantes de propagation de chacun de ces modes sont représentées sur la figure 6 pour le nickel à $24 \mathrm{GHz}$ et à $300 \mathrm{~K}$.
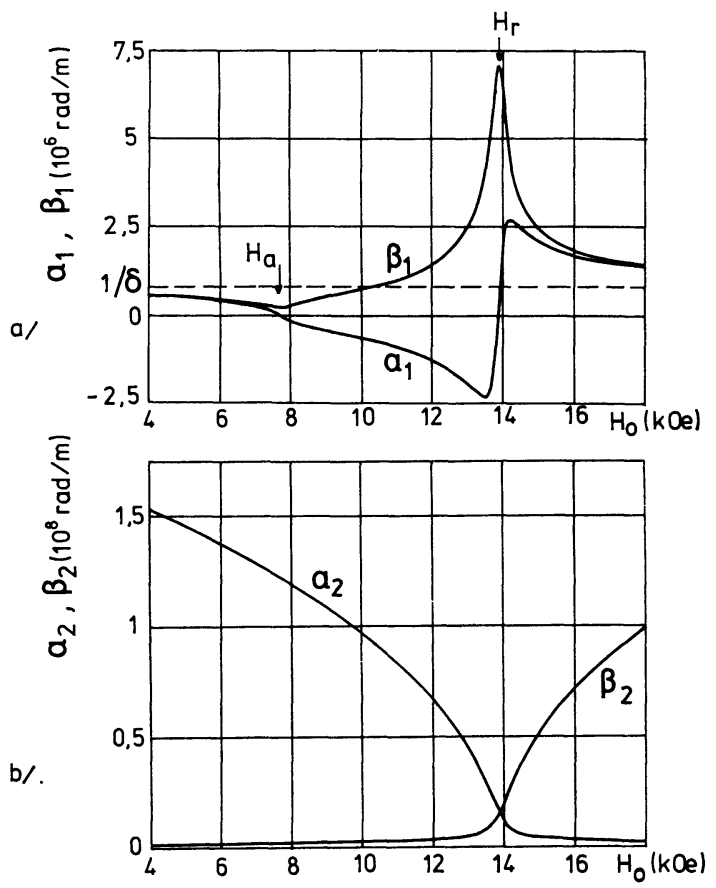

FIG. 6. - Variations théoriques des constantes de phase $\alpha_{i}$ et des constantes d'atténuation $\beta_{i}$ des modes 1 et 2 pour le nickel à $24 \mathrm{GHz}$ et $300 \mathrm{~K}\left(M_{\mathrm{s}}=490\right.$ u.e.m. ; $A=0,9 \times 10^{-6} \mathrm{erg} / \mathrm{cm}$; $\gamma=1,96 \times 10^{7} \mathrm{~s}^{-1} \mathrm{Oe}^{-1} ; v_{\mathrm{e}}=8 \times 10^{13} \mathrm{~s}^{-1} ; v_{\mathrm{m}}=5 \times 10^{9} \mathrm{~s}^{-1}$. a) $i=1$; b) $i=2$.

[Theoretical variations of phase constants $\alpha_{1}$ and $\alpha_{2}$ and attenuation constants $\beta_{1}$ and $\beta_{2}$ of modes 1 and 2 in nickel at $24 \mathrm{GHz}$ and $300 \mathrm{~K}$.

Ces courbes ont été tracées en prenant les valeurs suivantes des paramètres :

$$
\begin{aligned}
M_{\mathrm{s}}= & 490 \text { u.e.m. } \\
A & =0,9 \times 10^{-6} \mathrm{erg} . \mathrm{cm}^{-1} \\
\gamma & =1,96 \times 10^{7} \mathrm{~s}^{-1} \mathrm{Oe}^{-1} \\
v_{\mathrm{e}}= & 8 \times 10^{13} \mathrm{~s}^{-1} \text { correspondant à une conducti- } \\
& \text { vité }: \sigma_{0}=\omega_{\mathrm{p}}^{2} \varepsilon_{0} / \gamma_{\mathrm{e}}=0,9 \times 10^{7} \Omega . \mathrm{m} \\
v_{\mathrm{m}}= & 5 \times 10^{9} \mathrm{~s}^{-1} .
\end{aligned}
$$

Certaines de ces valeurs ont été déterminées après confrontation avec des expériences effectuées sur des lames de nickel [27].

Si $\eta$ est nul, les solutions de l'équation de dispersion (30) deviennent : $k_{1}=k_{\mathrm{e}}=(1+i) / \delta$ et $k_{2}=k_{\mathrm{m}}$.

Les constantes de propagation correspondant à $k_{\mathrm{e}}$ sont tracées en traits discontinus sur la figure $6 a$. On remarque que le mode 1 diffère considérablement de l'effet de peau ordinaire contrairement à ce qui est généralement admis.

Par contre, les effets de conduction n'ont pratiquement pas d'influence sur la loi de dispersion des ondes de spin. Cette dernière constatation a déjà été faite par Seavey et Tannenwald [28] ainsi que par Kittel [29] et Pincus [30]. Ces auteurs écrivaient l'équation de dispersion des ondes de spin modifiées par la conductivité :

$$
\omega=\omega_{\mathrm{r}}+\omega_{\mathrm{ech}} a^{2} k^{2}+\frac{\omega_{M}}{1+j \frac{k^{2} \delta^{2}}{2}} .
$$

Cette expression s'obtient d'ailleurs à partir de l'équation (33) moyennant quelques approximations [27].

3.1.3 Répartition du champ électromagnétique dans une lame. - Kittel [31] proposa d'exciter des ondes de spin stationnaires entre les deux faces parallèles d'une lame mince, par un champ magnétique alternatif uniforme sur l'épaisseur des échantillons. En fait, dans les expériences effectuées sur des matériaux conducteurs, l'onde électromagnétique qui arrive en incidence normale excite non seulement les ondes de spin mais aussi les oscillations élémentaires dues aux porteurs de charges électriques (effet de peau ordinaire à température ambiante). Il s'ensuit un couplage entre ces deux types d'oscillations et les phénomènes observés sur le taux de transmission ou l'impédance de surface ne sont pas dus à la propagation d'ondes de spin stationnaires pures.

Si l'onde incidente est polarisée circulairement à droite, elle excite deux ondes progressives $(+)$ correspondant aux modes 1 et 2 . Dans le cas d'une lame mince, ces deux ondes donnent naissance à deux ondes régressives $(-)$ par réflexion sur la face arrière de la lame. Si l'onde incidente est polarisée rectilignement, il faut associer à ces quatre ondes les quatre autres ondes à polarisation circulaire gauche. Nous ne traiterons par la suite que les modes à polarisation droite car les modes à polarisation gauche présentent peu d'intérêt lorsque le champ $\mathbf{H}_{0}$ varie.

Afin de pouvoir définir des impédances d'onde scalaires pour chacun des modes transversaux polarisés à droite, nous leur associerons des champs rotatifs [5] définis par :

$$
\varepsilon=E_{x}-j E_{y} ; \quad \mathscr{H}=j\left(H_{x}-j H_{y}\right) .
$$

La multiplication par $j$ dans l'expression du champ $\mathscr{H}$ traduit la quadrature dans l'espace des champs électriques et magnétiques.

Les champs dans la lame s'écrivent alors sous la forme :

$$
\varepsilon(z)=\varepsilon_{1}^{+} \mathrm{e}^{j k_{1 z}}+\varepsilon_{1}^{-} \mathrm{e}^{-j k_{1 z}}+\varepsilon_{2}^{+} \mathrm{e}^{j k_{2 z}}+\mathcal{E}_{2}^{-} \mathrm{e}^{-j k_{2 z}}
$$

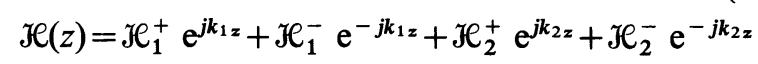

$$
\mathcal{M}(z)=\mathcal{M}_{1}^{+} \mathrm{e}^{j k_{1 z}}+\mathcal{M}_{1}^{-} \mathrm{e}^{-j k_{1 z}}+\mathcal{M}_{2}^{+} \mathrm{e}^{j k_{2 z}}+\mathcal{M}_{2}^{-} \mathrm{e}^{-j k_{2 z}} .
$$


Pour résoudre ces équations, nous devons prendre en compte les différentes grandeurs suivantes [27] :

- l'impédance d'onde $Z_{i}$ du mode $i$ :

$$
\dot{Z}_{i}=\varepsilon_{i}^{+} / \mathscr{H}_{i}^{+}=-\varepsilon_{i}^{-} / \mathscr{H}_{i}^{-}
$$

s'écrit aussi sous la forme plus générale :

$$
Z_{i}=\sqrt{\mu_{\mathrm{eff}}\left(k_{i}\right) / \varepsilon_{\mathrm{eff}}\left(k_{i}\right)} .
$$

et dans le cas qui nous intéresse : $Z_{i}=\omega \mu_{0} k_{i} / k_{\mathrm{e}}^{2}$;

- la susceptibilité $\chi_{i}$ du mode $i$ :

$$
\chi_{i}=\mathcal{M}_{i}^{+} / \mathscr{H}_{i}^{+}=\mathcal{M}_{i}^{-} / \mathscr{H}_{i}^{-}
$$

qui, pour les modes droits, s'écrit :

$$
\chi_{i}=\frac{\omega_{M}}{\omega_{\mathrm{r}}+\omega_{\mathrm{ech}} a^{2} k_{i}^{2}+j v_{\mathrm{m}}-\omega} .
$$

$$
\begin{aligned}
& \mathfrak{H}_{0}^{+}+\mathfrak{H}_{0}^{-}=\mathcal{H}_{1}^{+}+\mathcal{H}_{1}^{-}+\mathcal{H}_{2}^{+}+\mathcal{H}_{2}^{-} \\
& Z_{0}\left(\mathscr{H}_{0}^{+}-\mathscr{H}_{0}^{-}\right)=Z_{1}\left(\mathscr{H}_{1}^{+}-\mathscr{H}_{1}^{-}\right)+Z_{2}\left(\mathscr{H}_{2}^{+}-\mathscr{H}_{2}^{-}\right) \\
& 0=\chi_{1}\left(\left(\mathscr{H}_{1}^{+}+\mathscr{H}_{1}^{-}\right) \zeta+j k_{1}\left(\mathscr{H}_{1}^{+}-\mathscr{H}_{1}^{-}\right)\right)+\chi_{2}\left(\left(\mathscr{H}_{2}^{+}+\mathscr{H}_{2}^{-}\right) \zeta+j k_{2}\left(\mathscr{H}_{2}^{+}-\mathscr{H}_{2}^{-}\right)\right)
\end{aligned}
$$

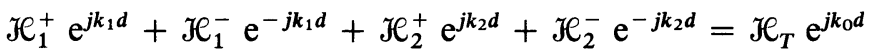

$$
\begin{aligned}
& Z_{1}\left(\mathscr{H}_{1}^{+} \mathrm{e}^{j k_{1} d}-\mathscr{H}_{1}^{-} \mathrm{e}^{-j k_{1} d}\right)+Z_{2}\left(\mathcal{H}_{2}^{+} \mathrm{e}^{j k_{2} d}-\mathscr{H}_{2}^{-} \mathrm{e}^{-j k_{2} d}\right)=Z_{0}^{\prime} \mathscr{H}_{T} \mathrm{e}^{j k_{0} d}
\end{aligned}
$$

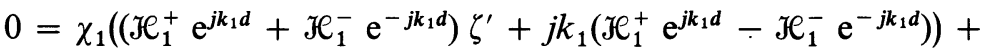

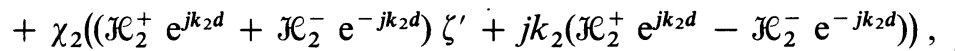

$Z_{0}$ et $Z_{0}^{\prime}$ étant respectivement les impédances d'onde des milieux situés devant et derrière la lame.

Quand il y a propagation d'ondes électromagnétiques dans le milieu, le champ électrique possède une composante tangentielle $E_{\alpha}(0)$ non nulle dans le plan $x \mathrm{O} y$ ( $\alpha$ pouvant être $x$ ou $y$ ). L'impédance de surface du matériau est alors par définition :

$$
Z_{\alpha \beta}=\frac{E_{\alpha}(0)}{I_{\beta}} \text { avec } I_{\beta}=\int_{0}^{\infty} J_{\beta}(z) \mathrm{d} z .
$$

$J$ est le courant qui traverse une section unitaire d'échantillon dans un plan perpendiculaire à la surface du matériau. On peut l'exprimer ên fonction des composantes du champ magnétique. On obtient alors :

$$
\left|\begin{array}{ll}
Z_{x x} & Z_{x y} \\
Z_{y x} & Z_{y y}
\end{array}\right|=\left|\begin{array}{cc}
\frac{E_{x}(0)}{H_{y}(0)} & -\frac{E_{x}(0)}{H_{x}(0)} \\
\frac{E_{y}(0)}{H_{y}(0)} & -\frac{E_{y}(0)}{H_{x}(0)}
\end{array}\right| .
$$

Pour des modes à polarisation circulaire :

$$
Z_{x x}=Z_{y y} \text { et } Z_{x y}=-Z_{y x} .
$$

L'impédance de surface des modes à polarisation circulaire droite peut alors s'exprimer par le rapport des champs définis en (35), soit :

$$
Z_{\mathrm{s}}=\frac{\mathcal{E}(0)}{\mathcal{H}(0)}=\frac{Z_{x x}+j Z_{x y}}{2} .
$$

La résolution du système d'équation (38) permet de calculer ce rapport ainsi que le taux de transmission défini par :

$$
T=\left|\frac{\mathscr{H}_{T}}{\mathfrak{H}_{0}^{+}}\right| .
$$

C'est par l'intermédiaire de ces deux grandeurs que l'on peut confronter la théorie et l'expérience. Nous allons décrire brièvement les phénomènes physiques correspondant aux résonances observées à température ambiante.

3.1.4 Résonance ferromagnétique. - A partir d'une certaine épaisseur (de l'ordre du micron pour le nickel et le permalloy), les ondes régressives sont trop atténuées et ne jouent plus aucun rôle. La seule singularité qui apparaît sur l'impédance de surface est la résonance ferromagnétique. Le système d'équations (38) a été résolu numériquement et nous avons représenté sur la figure 7 les variations théoriques de la partie réelle $R_{\mathrm{s}}$ et de la partie imaginaire $X_{\mathrm{s}}$ de l'impédance de surface d'une lame relativement épaisse de nickel à $24 \mathrm{GHz}$. Les calculs ont été faits avec les valeurs des paramètres données au paragraphe 3.1.2. Les courbes tracées correspondent aux deux valeurs extrêmes du blocage des spins en surface $\mathcal{M}=0$ et $\partial \mathcal{M} / \partial z=0$. Il apparaît clairement que les conditions aux limites sur l'aimantation en $z=0$ n'ont pratiquement aucune influence sur la variation de l'impédance de surface d'une telle lame. Lorsque ce blocage est complet $(\cdot k=0)$, l'impé- 


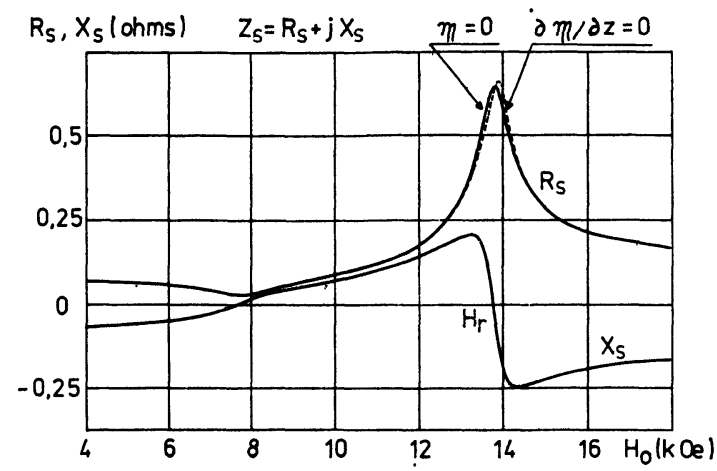

Fig. 7. - Variation de la partie réelle $R_{\mathrm{s}}$ et de la partie imaginaire $X_{\mathrm{s}}$ de l'impédance de surface d'une lame épaisse de nickel à $24 \mathrm{GHz}$. $\left(M_{\mathrm{s}}=490\right.$ u.e.m. ; $A=0,9 \times 10^{-6} \mathrm{erg} / \mathrm{cm} ; \gamma=1,96 \times 10^{7} \mathrm{rad}(\mathrm{s})^{-1}$ $(\mathrm{Oe})^{-1} ; v_{\mathrm{c}}=8 \times 10^{13} \mathrm{~s}^{-1} ; v_{\mathrm{m}}=5 \times 10^{9} \mathrm{~s}^{-1}$.

[Variations of the real part $R_{\mathrm{s}}$ and the imaginary part $X_{\mathrm{s}}$ of the surface impedance for a thick nickel plate at $24 \mathrm{GHz}$.]

dance de surface $Z_{\mathrm{s}}$ peut s'exprimer analytiquement par une relation simple qui fait intervenir les impédances d'onde $Z_{1}$ et $Z_{2}$ de chacun des modes [27].

Le champ électromagnétique dans la lame est la superposition des champs dus à ces deux modes dont les constantes de propagation ne sont pas nulles à la résonance ferromagnétique. Celle-ci ne peut donc pas correspondre à la précession uniforme des spins. Pour que l'aimantation alternative et le champ d'excitation hyperfréquence soient parallèles, il faudrait que les pertes et les termes d'échange soient nuls.

3.1.5 Ondes de spin stationnaires. - Pour un champ magnétique inférieur au champ de résonance ferromagnétique $H_{\mathrm{r}}$, le mode 2 présente peu de pertes (Fig. 6b). Pour des lames de faible épaisseur, il peut s'établir un régime d'ondes stationnaires à l'intérieur de celle-ci. La théorie classique ne donne que les champs de résonance alors que la structure du champ électromagnétique dans la lame permet de déterminer ces champs ainsi que la forme exacte des raies observées.

a) Théorie classique. - L'aimantation est généralement prise sous la forme :

$$
\mathcal{M}(z)=\mathcal{M}^{+} \mathrm{e}^{j k z}+\mathcal{M}^{-} \mathrm{e}^{-j k z}
$$

où $k$ correspond sensiblement au vecteur d'onde $k_{2}$ de notre modèle. Le mode électrocinétique est alors assimilé à l'effet de peau ordinaire. En reportant cette égalité dans les équations (37), on trouve la relation de dispersion des ondes de spin stationnaires [34] :

$$
\left(\zeta \zeta^{\prime}+k^{2}\right) \operatorname{tg} k d=k\left(\zeta^{\prime}-\zeta\right) .
$$

A partir de cette théorie, plusieurs hypothèses ont été avancées sur les valeurs et signes respectifs de $\zeta$ et $\zeta^{\prime}$ pour retrouver les champs de résonance expérimentaux $[34,35]$. b) Théorie des modes d'ondes de spin. - Pour calculer l'impédance de surface $Z_{\mathrm{s}}$ d'une lame mince, la seule méthode rigoureuse consiste à résoudre numériquement le système d'équations (38). Nous donnons, à titre indicatif, sur la figure 8 , les variations de la partie réelle de $Z_{\mathrm{s}}$ de lames minces de nickel dans le cas d'un fort blocage des spins en surface (condition de Kittel : $\mathcal{M}=0$ ).

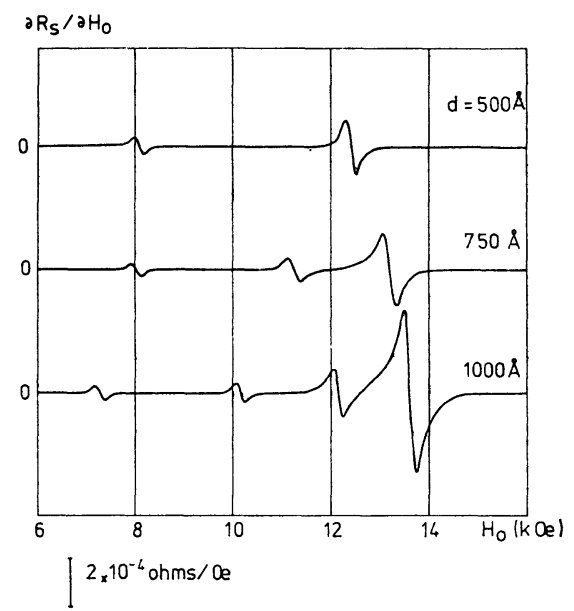

FIG. 8. - Variations théoriques de la dérivée de la résistance de surface $R_{\mathrm{s}}$ de lames minces de nickel à température ambiante pour un blocage complet des spins en surface $(\mathcal{H}=0)$; les paramètres sont ceux de la figure 7 .

[Theoretical variations of the derivative of the surface resistance $R_{\mathrm{s}}$ for thin nickel plates at room temperature and for a strong pinning, the parameters are those of figure 7.$]$

Les résultats expérimentaux dans le nickel font apparaître un nombre de raies plus faibles et de formes différentes. De nombreux travaux ont été publiés pour tenter d'expliquer les divergences sur l'emplacement des raies. La figure 9 montre quelquesuns des résultats expérimentaux obtenus avec des lames polycristallines de nickel.

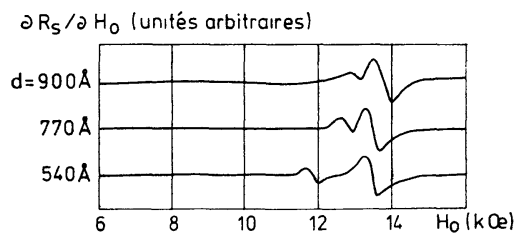

FIG. 9. - Résultats expérimentaux obtenus avec des lames minces polycristallines de nickel à $24 \mathrm{GHz}$ et à température ambiante. [Experimental results obtained with thin polycrystalline nickel plates at $24 \mathrm{GHz}$ and at room temperature.]

Nous avons pu retrouver théoriquement ces courbes (Fig. 10) en faisant intervenir un blocage partiel des spins en surface, traduit par une constante d'anisotropie de surface : $K_{\mathrm{S}}=0,2 \mathrm{erg} / \mathrm{cm}^{2}$ qui se situe dans la plage des valeurs prévues par Neel [33] $\left(K_{\mathrm{S}}=|\zeta| A\right)$.

Les courbes théoriques et expérimentales se superposent lorsque les épaisseurs théoriques sont légè- 


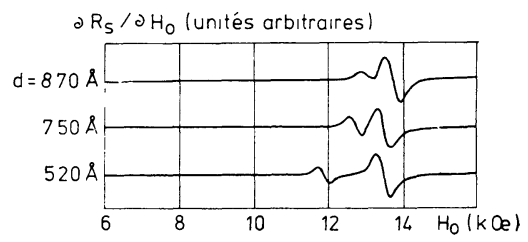

FIG. 10. - Résultats théoriques obtenus avec des lames minces de nickel à partir du model à deux modes couplés $\left(v_{\mathrm{e}}=8 \times 10^{13} \mathrm{~s}^{-1}\right.$; $v_{\mathrm{m}}=5 \times 10^{9} \mathrm{~s}^{-1} ; A=0,9 \times 10^{-6} \mathrm{erg} / \mathrm{cm} ; K_{\mathrm{s}}=0,2 \mathrm{erg} / \mathrm{cm}^{2}$ : $f=24 \mathrm{GHz} ; T=300 \mathrm{~K})$

[Theoretical results obtained with thin nickel plates from the pattern with two coupled modes.]

rement inférieures (de 20 à $30 \AA$ ) à celles mesurées. Ceci peut s'expliquer par l'existence d'une couche superficielle sur les faces de la lame ne possédant pas les mêmes propriétés que le cœur du matériau.

Une discussion plus approfondie du rôle et des signes des constantes d'anisotropie de surface sur chaque face de la lame a été publiée par ailleurs [36].

3.1.6 Transmission autour de l'antirésonance. Pour des lames minces, on n'observe aucune variation de l'impédance de surface autour de l'antirésonance, aussi bien expérimentalement que théoriquement, quel que soit le pinning choisi. Pour des épaisseurs voisines de $1000 \AA$, le facteur de transmission ne présente pas de sıngularités près de l'antirésonance qui, dans le nickel à $24 \mathrm{GHz}$, se produit pour un champ magnétique de 7700 Oe. Les seules variations de la transmission observées expérimentalement sont dues à la résonance ferromagnétique et aux ondes de spin stationnaires [36].

Lorsque l'épaisseur des lames augmente, les ondes stationnaires disparaissent. Heinrich et al. [37, 38], décelèrent un accroissement du taux de transmission à l'antirésonance dans des lames relativement épaisses $(\simeq 10 \mu \mathrm{m})$. Ces auteurs attribuent cette particularité à une modulation de l'épaisseur de peau

$$
\delta=\sqrt{2 / \sigma_{0} \mu_{\text {eff }} \omega}
$$

par les variations de la perméabilité effective $\mu_{\text {eff }}$ qui passe par un minimum à l'antirésonance.

Le facteur de transmission $T$ défini en (39b) se calcule rigoureusement en partant de la structure du champ électromagnétique dans la lame que nous avons déterminée à partir des modes propres (Eq. (38)). La figure 11 représente les variations de $T$ pour une lame de nickel de $10 \mu \mathrm{m}$ à $24 \mathrm{GHz}$.

Au champ d'antirésonance, la puissance transmise par cette lame est environ 1000 fois supérieure à celle transmise en l'absence de champ magnétique statique $H_{0}$.

Nous avons montré qu'autour du champ d'antirésonance $H_{\mathrm{a}}$, le mode 1 est prépondérant [2], on peut donc donner une expression analytique du facteur de transmission en ne tenant compte que de ce mode. Cette hypothèse - qui a servi de base aux calculs de plusieurs auteurs [37-39] — revient en fait à négliger la dispersion spatiale dans la perméabilité.

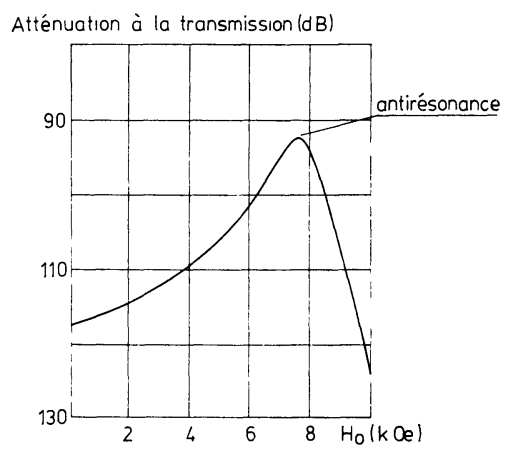

Fig. 11. - Transmission théorique à $24 \mathrm{GHz}$ à travers un échantillon de $10 \mu \mathrm{m}$. Les valeurs des paramètres sont celles de la figure 7 .

[Theoretical transmission at $24 \mathrm{GHz}$ through a sample $10 \mu \mathrm{m}$ thick. The parameter values are given in figure 7.$]$

Le vecteur d'onde $k_{1}$ du mode 1 déterminé à partir de (32) s'écrit :

$$
k_{1}^{2} \simeq k_{\mathrm{e}}^{2}(1-\eta) \simeq k_{\mathrm{e}}^{2}\left(\frac{\omega-\omega_{\mathrm{a}}+j v_{\mathrm{m}}}{\omega_{M}}\right) .
$$

Lorsque le champ magnétique varie au voisinage de $H_{\mathrm{a}}$, les variations des constantes de propagation dépendent principalement de $v_{\mathrm{m}}$. L'antirésonance a donc été proposée pour mesurer ce paramètre. Celui-ci est habituellement déterminé à partir de la largeur de la raie de résonance ferromagnétique où les modes 1 et 2 interviennent, ce qui complique l'interprétation des résultats expérimentaux dans le cas des conducteurs ferromagnétiques.

3.2 COUPlage DES ONDES DE Plasma ET DES ONDES DE SPIN A BASSE TEMPÉRATURE DANS LE NICKEL. 3.2.1 Résultats expérimentaux. - Nous avons déjà signalé qu'à température ordinaire, on n'observe rien par réflexion autour de l'antirésonance. $\mathrm{Au}$ contraire, lorsque la température diminue, l'impédance de surface d'une lame monocristalline de nickel taillée dans le plan [100] présente une singularité près du champ d'antirésonance (Fig. 12). Celui-ci est plus grand qu'à température ordinaire, du fait de l'influence de l'anisotropie magnétocristalline.

Le moac̀le théorique à deux modes décrit dans la figure 12 ne permet pas de retrouver ces singularités sur l'impédance de surface quelles que soient les variations des paramètres adoptés. Nous sommes donc amenés à préciser le rôle de la température sur la perméabilité et la conductivité.

3.2.2 Conductivité et perméabilité à basse température. - Dans l'équation du mouvement de l'aimantation, nous avons négligé jusqu'ici l'influence des anisotropies magnétocristalline et magnétostrictive. A basse température, cette hypothèse n'est plus valable. Pour des échantillons monocristallins de nickel taillés dans des plans [100] ou [111], ces effets se traduisent par un champ effectif supplémentaire qu'on peut inclure dans l'expression de $\omega_{r}$ [2]. Les coefficients du tenseur de perméabilité conservent 

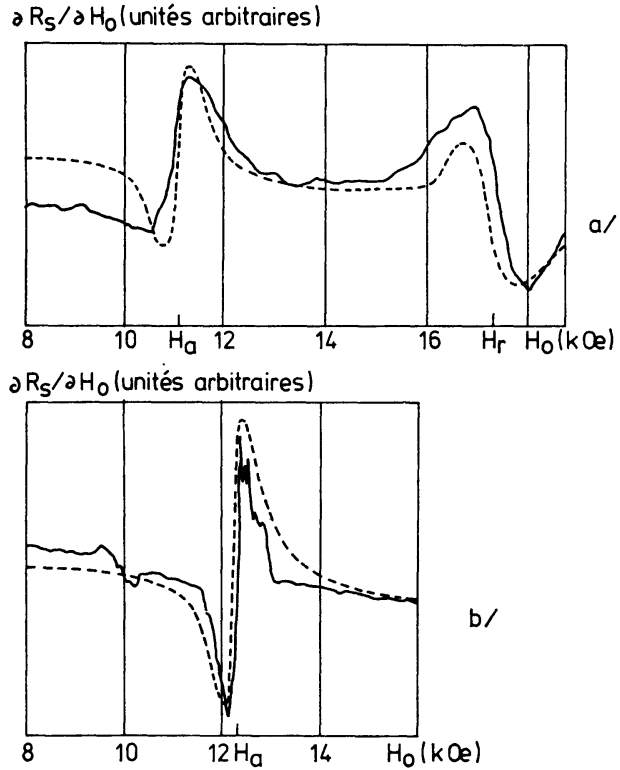

Fig. 12. - Variations expérimentale (en trait plein) et théorique (en trait interrompu) de la dérivée de la résistance de surface d'une lame monocristalline de nickel [100] d'épaisseur $5 \mu \mathrm{m}(f=24 \mathrm{GHz})$; a) $T=77 \mathrm{~K} ; b) T=2 \mathrm{~K}$.

[Experimental variations and theoretical variations of the surface resistance for a monocrystalline nickel plate [100] $5 \mu \mathrm{m}$ thick $(f=24 \mathrm{GHz})$.]

alors la forme donnée au paragraphe 1 , les effets de la température ne se manifestant que par une modification des fréquences caractéristiques, ce qui n'apporte aucun phénomène nouveau.

L'influence de la température sur la conductivité est plus complexe et nous n'envisagerons que le cas d'un métal ferromagnétique présentant une surface de Fermi quasi sphérique. Lorsque la température diminue, le libre parcours moyen augmente et devient comparable à la profondeur de pénétration de l'onde. Certains porteurs ont une composante de vitesse suivant la direction de propagation voisine de la vitesse de phase des ondes : il s'ensuit une interaction onde-porteurs. C'est un phénomène analogue à l'effet Doppler et ces porteurs ont été appelés dopplerons par Konstantinov et Skobov [40].

Le tenseur de conductivité, calculé à partir de l'équation de Boltzmann, dépend alors du vecteur d'onde $\mathbf{k}$ (dispersion spatiale) par l'intermédiaire d'un terme $\xi$ donné par la relation suivante $[2,5]$ :

$$
\xi=\frac{\omega-\omega_{\mathrm{c}}+j v_{\mathrm{e}}}{k v_{\mathrm{F}}}
$$

où $v_{\mathrm{F}}$ est la vitesse de Fermi des porteurs.

3.2.3 Equation de dispersion à basse température. Lorsque le champ magnétique statique est appliqué perpendiculairement à l'échantillon, l'équation $(17 b)$ des ondes droites qui se propagent à basse température s'écrit :

$$
\begin{aligned}
k^{2}= & \frac{3}{4} \frac{\omega \cdot \omega_{\mathrm{p}}^{2}}{c^{2} k v_{\mathrm{F}}}\left(\left(1-\xi^{2}\right) \ln \frac{\xi-1}{\xi+1}-2 \xi\right) \\
& \times\left(\frac{\omega_{\mathrm{a}}-\omega+\omega_{\mathrm{ech}} a^{2} k^{2}-j v_{\mathrm{m}}}{\omega_{\mathrm{r}}-\omega+\omega_{\mathrm{ech}} a^{2} k^{2}-j v_{\mathrm{m}}}\right) .
\end{aligned}
$$

A température ordinaire, $\xi \gg 1$ et à partir de l'équation ci-dessus, on retrouve l'équation de dispersion (26). A très basse température, $\xi \ll 1$, les collisions n'interviennent plus. Si l'on considère un métal fictif ayant les mêmes propriétés de conduction que le métal ferromagnétique, mais avec une perméabilité effective égale à 1 ; l'équation (44) donne le vecteur d'onde $k_{\mathrm{e}}^{\prime}$ de l'effet de peau extrêmement anormal :

$$
k_{\mathrm{e}}^{\prime 3}=\frac{3 \pi}{4 j} \frac{\omega_{\mathrm{p}}^{2}}{c^{2}} \frac{\omega}{v_{\mathrm{F}}}
$$

La relation (44) montre qu'à l'antirésonance, $k$ est très faible. L'effet de peau anormal apparaît lorsque le produit $k l \gtrsim 1$. Donc, dans un métal ferromagnétique à l'antirésonance, le seuil d'apparition de l'effet de peau anormal est repoussé par rapport à un métal ordinaire de même conductivité. Cette nouvelle situation privilégiée de l'antirésonance a été déjà signalée par Kaganov [41].

L'équation (44) admet trois solutions [2]. Le mode 1 de la température ordinaire s'est dédoublé en deux modes que nous appellerons 1 et 3 . Sur les figures $13 a$ et $13 \mathrm{~b}$, nous donnons les constantes de propagation de ces modes à $24 \mathrm{GHz}$ pour le nickel à une température voisine de $2 \mathrm{~K}$.
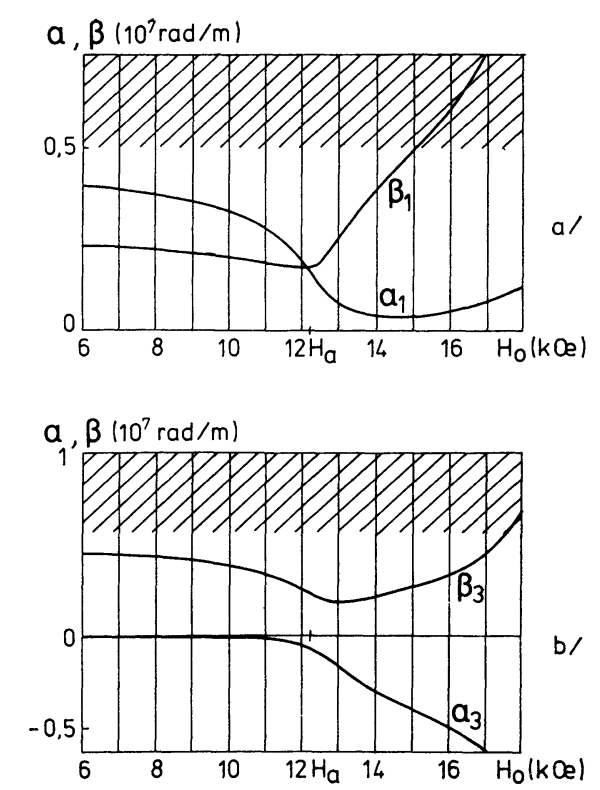

Fig. 13. - Constantes de propagation des modes 1 (Fig. 13a) et 3 (Fig. 13b) dans le nickel $\left(f=24 \mathrm{GHz} ; T=2 \mathrm{~K} ; v_{\mathrm{e}}=10^{11} \mathrm{~s}^{-1}\right.$; $\left.\left.v_{\mathrm{m}}=7 \times 10^{9} \mathrm{~s}^{-1} ; 2\left|K_{1} / M_{\mathrm{s}}\right|=4810 \mathrm{Oe}\right) ; a\right)$ mode $1 ;$ b) mode 3 .

[Propagation constants of mode 1 (Fig. 13a) and mode 3 (Fig. 13b) in nickel.] 
Sur ces figures, nous avons hachuré la zone où l'on doit tenir compte de l'effet de peau anormal $(k l \geqslant 1)$. Le mode 2 n'est pas représenté car il reste très proche d'une onde de spin pure.

3.2.4 Impédance de surface. - A température ordinaire, l'impédance de surface au voisinage de l'antirésonance est pratiquement égale à l'impédance d'onde du mode 1 , soit :

$$
Z_{\mathrm{s}} \simeq Z_{1}=\frac{\omega \mu_{0} \mu_{\mathrm{eff}}\left(k_{1}\right)}{k_{1}}
$$

Compte tenu de l'expression de $\mu_{\text {eff }}\left(k_{1}\right)$ qui apparaît dans l'équation (26), on peut écrire :

$$
Z_{\mathrm{s}} \simeq \omega \mu_{0} \frac{\omega-\omega_{\mathrm{a}}+j v_{\mathrm{m}}}{\omega_{M} k_{1}}
$$

En faisant intervenir l'épaisseur de peau classique $\delta$ et la valeur de $k_{1}$, on obtient :

$$
Z_{\mathrm{s}} \simeq \frac{\omega \mu_{0} \delta}{\sqrt{2}}\left(\frac{v_{\mathrm{m}}-j\left(\omega-\omega_{\mathrm{a}}\right)}{\omega_{M}}\right)^{1 / 2}
$$

$\mathrm{Si}$ on considère, à très basse température, le mode 1 toujours prépondérant autour de l'antirésonance, l'expression de $Z_{\mathrm{s}}$ devient :

$$
Z_{\mathrm{s}} \simeq \frac{\omega \mu_{0} \delta^{\prime}}{(3 / 4)^{1 / 3}}\left(\frac{\nu_{\mathrm{m}}+\left(\omega-\omega_{\mathrm{a}}\right)-j\left(\omega-\omega_{\mathrm{a}}-v_{\mathrm{m}}\right)}{\omega_{M}}\right)^{2 / 3}
$$

où $\delta^{\prime}$ est l'épaisseur effective de l'effet de peau anormal : $\delta^{\prime}=\left(\frac{l}{2 \pi \omega \mu_{0} \sigma_{0}}\right)^{1 / 3}$. On retrouve alors l'expression de l'impédance de surface donnée par ailleurs [41] qui fait apparaître une modification de l'exposant lorsque l'on passe de la température ordinaire à une température très basse.

En fait, si autour de l'antirésonance, l'influence du mode 2 peut être négligée, nous avons montré qu'il n'en est pas de même pour le mode 3 à basse température [2]. Dans ces conditions, seule l'étude de la répartition du champ électromagnétique dans la lame permet une interprétation rigoureuse des résultats expérimentaux. Nous supposerons la lame suffisamment épaisse pour que les ondes régressives réfléchies par la face arrière aient un rôle négligeable. Cette hypothèse est justifiée pour des échantillons de $5 \mu \mathrm{m}$ d'épaisseur utilisés pour obtenir les résultats expérimentaux de la figure 12. Le champ électromagnétique dans la lame résulte de la superposition des ondes progressives associées aux trois modes propres solutions de l'équation (44). L'apparition du mode 3 est due à la présence du terme $k v_{\mathrm{F}}$ dans le tenseur de permittivité, traduisant ainsi l'influence des dopplerons. Pour calculer théoriquement l'impédance de surface, il est nécessaire d'introduire une condition aux limites supplémentaire. Celle-ci est fournie par le type de réflexion des électrons sur les surfaces de séparation air-métal.

La conductivité du matériau se calcule à partir de la fonction de distribution des vitesses des électrons qui est composée de deux termes : $f_{0}$ qui dépend de l'énergie et $f_{1}$ qui traduit les perturbations dues à l'effet du champ électromagnétique. La réflexion diffuse est telle qu'après le choc avec la surface, un électron quitte celle-ci avec une fonction de distribution non perturbée $f_{0}$. La condition aux limites supplémentaire est alors obtenue en exprimant la densité de courant associée aux électrons réfléchis [2].

La résolution du système d'équations ainsi obtenu permet de retrouver théoriquement les variations expérimentales de l'impédance de surface autour de l'antirésonance. Ces résultats théoriques sont représentés en lignes discontinues sur la figure 12. Parmi tous les paramètres sur lesquels on peut agir pour obtenir un bon accord entre la théorie et l'expérience, c'est la fréquence de collisions $v_{\mathrm{e}}$ qui joue le rôle le plus important. Ainsi, à $77 \mathrm{~K}$, la valeur choisie correspond à une conductivité environ 20 fois plus forte qu'à la température ambiante. Les valeurs prises pour les champs effectifs traduisant l'effet de l'anisotropie magnétocristalline ont été obtenues à partir de mesures de couple [42]. Certains paramètres $\left(K_{\mathrm{s}}, A\right)$ ne jouent qu'un rôle secondaire sur les phénomènes observés par réflexion autour de l'antirésonance ; ils doivent donc être déterminés par d'autres expériences (exemple : ondes de spins stationnaires).

Conclusion. - Les différents auteurs qui ont constaté de nombreuses divergences entre les résultats théoriques et expérimentaux dans l'observation des résonances ferromagnétiques et des ondes de spin stationnaires les ont souvent attribuées à l'imperfection du modèle électromagnétique généralement utilisé. En effet, si les ondes électromagnétiques ont depuis longtemps constitué un moyen d'investigation des propriétés magnétiques des solides et, en particulier, des milieux conducteurs ferromagnétiques, la résolution complète des équations de Maxwell dans les milieux à perméabilité et conductivité tensorielles nous a amené à étudier le couplage entre les effets de conduction et les effets magnétiques sans admettre a priori que l'onde électrocinétique se réduit à l'effet de peau. Nous avons pu ainsi expliquer tous les phénomènes observés en réflexion et en transmission sur des échantillons de nickel aussi bien à température ordinaire qu'à basse température. La dispersion des ondes électromagnétiques résultant du couplage des ondes électrocinétiques et des ondes de spin dans les semi-conducteurs ferromagnétiques commence à être étudiée en vue de la réalisation de circuits hyperfréquences (lignes unidirectionnelles, amplificateurs, etc.). Devant les difficultés rencontrées actuellement pour obtenir des échantillons convenables de ces matériaux, on préfère s'orienter dans l'immédiat vers des structures mixtes ferrite-semiconducteur ordinaire qui peuvent être aussi étudiées à partir du modèle théorique proposé. 


\section{Bibliographie}

[1] LAX, B. et Button, K. J., Microwaves ferrites and ferrimagnetics (Mc Graw Hill, New York) 1962, a) p. 150 ; b) p. 423.

[2] Monllor, C., Couplage des ondes de plasma et des ondes de spin dans les conducteurs ferromagnétiques. Etude de l'antirésonance dans des lames de nickel. Thèse Doctorat ès-Sciences physiques, I.N.P. Grenoble (1976).

[3] BaR'yakhtaR, Y. G., Savchenko, M. A. and Stepanov, K. N., Sov. Phys. J.E.T.P. 23 (1966) 383.

[4] Steele, M. C. and Vural, B., Wave interactions in solid state plasmas (Mc Graw-Hill, New York) 1969.

[5] Kaner, E. A. and Sкobov, V. G., Plasma effects in metalshelicon and alfven waves (Monog. on Phys., Taylor and Francis, Londres) 1971.

[6] Konstantinov, O. V. and Perel, V. I., Sov. Phys. J.E.T.P. 11 (1960) 117.

[7] Veselago, V. G., Sov. Phys. Usp. 10 (1968) 509.

[8] Blank, A. Ya. and Kaganov, M. I., Sov. Phys. Usp. 10 (1968) 536.

[9] Ament, W. S. and Rado, G. T., Phys. Rev. 97 (1955) 1558.

[10] Hines, M. E., IEEE Trans Microwave Theory Techn. 19 (1971) 442.

[11] Courtois, L., Chiron, B. and Forterre, G., Câbles \& transm. 4 (1973) 416.

[12] Gerson, T. J. and Nadan, J. S., I.E.E.E. Trans. Microwave Theory Techn. 22 (1974) 757.

[13] Robinson, B. B., Vural, B. and Parekh, J. P., I.E.E.E. Trans. Electron Devices 17 (1970) 224.

[14] Yamada, S., Chang, N. S. and Matsuo, U., I.E.E.E. Trans. Microwave Theory Techn. 25 (1977) 600.

[15] Bini, M., Filetti, P. L., Millanta, L. and Rubino, N., $J$. Appl. Phys. 47 (1976) 3209.

[16] Dabydov, A. B., Zakharov, V. A. and Podchinenov, I. E., Sov. Phys. Solid State 19 (1977) 978.

[17] Eshbach, J. R. and Damon, R. W., Phys. Rev. 118 (1960) 1208.

[18] Marcoux, J., Etude en hyperfréquence d'un circulateur à lames minces semiconductrices polycristallines. Thèse Doctorat Spécialité, Université de Toulouse (1974).

[19] Luong, B., Etude en bande $Q$ d'un isolateur d'antimoniure d'indium. Rapport D.E.A., I.N.P. Grenoble (1976).

[20] Suzuki, K. and HiRota, R., I.E.E.E. Trans. Electron. Devices 18 (1971) 408.
[21] Lehmann, H. W. and Robbins, M., J. Appl. Phys. 37 (1966) 1389.

[22] Shafer, M. W. and McGuire, T. R., J. Appl. Phys. 39 (1968) 588.

[23] Kurinobu, S., Uchiyama, S., Fujil, T. and Yamada, K., Jpn. J. Appl. Phys. 15 (1976) 1073.

[24] Funahashi, S. and Kazama, N., J. Phys. Soc. Japan 41 (1976) 811.

[25] Monllor, C., Bouthinon, M. and Chilo, J., Proc. Int. Conf. Magn. Amsterdam (1976), Physica 86-88B (1977) 1247.

[26] Frait, Z., Ferromagnetic resonance in metals. Proc. Int. Conf: Magn., Moscou no 4 (1973), p. 16.

[27] Bouthinon, M., Modes d'ondes de spin dans les conducteurs ferromagnétiques. Application à l'étude de la propagation des ondes électromagnétiques dans le nickel. Thèse Doctorat ès-Sciences physiques, I.N.P. Grenoble (1974).

[28] Seavey, M. H. and Tannenwald, P. E., Phys. Rev. Lett. 1 (1958) 168.

[29] Kittel, C., Phys. Rev. 110 (1958) 836.

[30] Pincus, P., Phys. Rev. 118 (1960) 658.

[31] Kittel, C., Phys. Rev. 110 (1958) 1295.

[32] Rado, G. T. and Weertman, J. R., J. Phys. Chem. Solids 11 (1959) 315.

[33] Neel, L., J. Physique Radium 15 (1954) 225.

[34] Korchagin, Yu. A., Khlebopros, R. G. and ChistyaKov, N. S., Sov. Phys. Solid State 14 (1973) 1826.

[35] Sementsov, D. I., Sov. Phys. Solid State 16 (1974) 604.

[36] Monllor, C., Bouthinon, M. and Baribaud, M., Phys. Status Solidi (a) 29 (1975) 481

[37] Heinrich, B. and Meshcheryakov, V. F., Sov. Phys. J.E.T.P. 32 (1971) 232.

[38] Dewar, G., Heinrich, B. and Cochran, J. F., Ferromagnetic antiresonance transmission of $24 \mathrm{GHz}$ radiation through nickel. Can. J. Phys., à paraître (1977).

[39] Kaganov, M. I. and Mikhailov, A. S., Sov. Phys. Solid State 14 (1973) 2748.

[40] Konstantinov, O. V. and Skobov, V. G., Sov. Phys. J.E.T.P. 34 (1972) 885.

[41] Kaganov, M. I. and PaAsch, G., Sov. Phys. J.E.T.P. 43 (1976) 580.

[42] EsCUDIER, P., L'anisotropie de l'aimantation : un paramètre important de l'étude de l'anisotropie magnétocristalline. Thèse Doctorat ès-Sciences physiques, Grenoble (1973). 